San Jose State University

SJSU ScholarWorks

Master's Theses

Master's Theses and Graduate Research

1990

\title{
Numerical methods for solving transient heat conduction problems with combined convection and radiation
}

Lap Yin Stephen $\mathrm{Ng}$

San Jose State University

Follow this and additional works at: https://scholarworks.sjsu.edu/etd_theses

\section{Recommended Citation}

$\mathrm{Ng}$, Lap Yin Stephen, "Numerical methods for solving transient heat conduction problems with combined convection and radiation" (1990). Master's Theses. 3339.

DOI: https://doi.org/10.31979/etd.wgm8-ahtn

https://scholarworks.sjsu.edu/etd_theses/3339

This Thesis is brought to you for free and open access by the Master's Theses and Graduate Research at SJSU ScholarWorks. It has been accepted for inclusion in Master's Theses by an authorized administrator of SJSU ScholarWorks. For more information, please contact scholarworks@sjsu.edu. 


\section{INFORMATION TO USERS}

The most advanced technology has been used to photograph and reproduce this manuscript from the microfilm master. UMI films the text directly from the original or copy submitted. Thus, some thesis and dissertation copies are in typewriter face, while others may be from any type of computer printer.

The quality of this reproduction is dependent upon the quality of the copy submitted. Broken or indistinct print, colored or poor quality illustrations and photographs, print bleedthrough, substandard margins, and improper alignment can adversely affect reproduction.

In the unlikely event that the author did not send UMI a complete manuscript and there are missing pages, these will be noted. Also, if unauthorized copyright material had to be removed, a note will indicate the deletion.

Oversize materials (e.g., maps, drawings, charts) are reproduced by sectioning the original, beginning at the upper left-hand corner and continuing from left to right in equal sections with small overlaps. Each original is also photographed in one exposure and is included in reduced form at the back of the book.

Photographs included in the original manuscript have been reproduced xerographically in this copy. Higher quality 6" x 9 " black and white photographic prints are available for any photographs or illustrations appearing in this copy for an additional charge. Contact UMI directly to order.

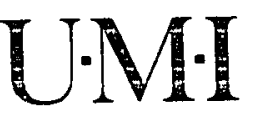

University Microfilms International

A Bell \& Howell Information Company 300 North Zeeb Road. Ann Arbor, MI 48106-1346 USA

$313: 761.4700 \quad 800: 521.0600$ 

Order Number 1941690

Numerical methods for solving transient heat conduction problems with combined convection and radiation

Ng, Lap Yin Stephen, M.S.

San Jose State University, 1990 


\section{.}




\title{
NUMERICAL METHODS FOR SOLVING TRANSIENT HEAT CONDUCTION PROBLEMS WITH COMBINED CONVECTION AND RADIATION
}

\author{
A Thesis \\ Presented to \\ The Facuity of the Depâriment of Mathematics \\ San Jose State University
}

\author{
In Partial Fulfillment \\ of the Requirements for the Degree \\ Master of Science
}

By

Lap Yin Stephen $\mathrm{Ng}$

August, 1990 
Approved for the Department of Mathematics

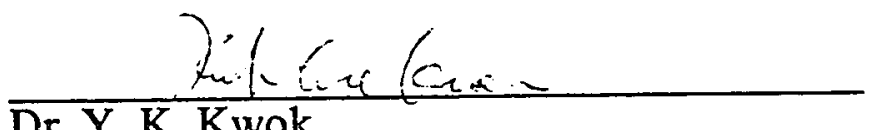

Dr. Y. K. Kwok

Samih Obad

Dr. Samih Obaid

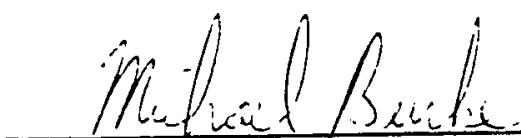

Dr. Michael Burke

Approved for the University

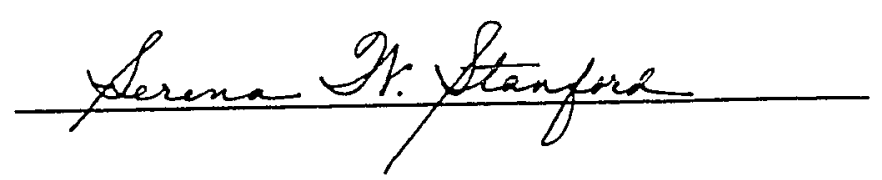




\section{ABSTRACT \\ NUMERICAL METHODS FOR SOLVING TRANSIENT HEAT CONDUCTION PROBLEMS WITH COMBINED CONVECTION AND RADIATION by Lap Yin Stephen $\mathrm{Ng}$}

The transient surface temperature distribution is determined for the flat plate and sphere subjected to cooling by combined convection and radiation. In the study, the initial-boundary value problem is reduced to a singular nonlinear Volterra integral equation of the second kind using the integral transform method. Three numerical techniques are introduced in an attempt to find an approximated solution of the problem. The integral equation is solved numerically on a computer by the Runge-Kutta method of orders 1, 3, and 5. In addition, the finite difference method is implemented to solve the initial-boundary value problem, and the solutions are compared with those generated by the Runge-Kutta Method. All the numerical results are presented graphically. Limitations and difficulties involved in these schemes are discussed. At the end, a numerical algorithm for solving the problem is proposed. 
Dedicated to

Anna, Ida, Regina(Rebecca), and Maraina. 


\section{ACKNOWLEDGMENTS}

This thesis would not have been possible without the assistance of many people. I would like to thank my advisor, Dr. Yue-Kuen Kwok, for his patience and guidance throughou the making of the thesis. His helpful and generous advice and suggestions have greatly improved my work. Thanks, also, to the rest of my committee, Dr. Samih Obaid and Dr. Micheal Burke. I gratefully acknowledge the fine work of William Roleston and Robert Supencheck who helped with the proofreading. Deep appreciation is extended to Yvonne Ho. Without her prayer, I never would have made it to this point. Especially to my family, thank you for all of their encouragement. Lastly, I express my deepest appreciation to my Heavenly Father for His severe grace during the preparation of this thesis. 


\section{TABLE OF CONTENTS}

Page

$\mathbf{v}$

viii

LIST OF TABLES AND FIGURES

Chapter

I. ANALYTIC SOLUTIONS OF THE HEAT EQUATION SUBJECTED TO COMBINED CONVECTIVE AND RADIATIVE BOUNDARY CONDITIONS 1

1.1 Introduction 1

1.2 Statement of the Problem 5

1.3 The Laplace Transform Method 6

1.4 The Eigenvalue Expansion Method 17

1.5 Remarks 24

II. THE METHOD OF SUCCESSIVE APPROXIMATIONS 26

2.1 Introduction 26

2.2 Outline of the Method 27

III. THE RUNGE-KUTTA METHOD 30

3.1 Introduction 30

3.2 Outline of the Method 31

3.3 The Third Order Approximation 47

3.4 The Fifth Order Approximation 49

3.5 Remarks 53

IV. THE FINITE DIFFERENCE METHOD 54

4.1 Introduction 54

4.2 Crank-Nicolson Scheme 55

vi 
4.3 Two Special Cases 60

4.3.1 The Flat Plate 60

4.3.2 The Sphere 62

4.4 Stability 64

4.5 Refinement of a Partition and Extrapolation Techniques 66

V. NUMERICAL RESULTS 69

5.1 Introduction 69

5.2 Results for the Flat Plate and the Sphere 70

VI. CONCLUSION 79

REFERENCES $\quad 83$

APPENDIX $\quad 85$ 


\section{List of Tables and Figures}

Table

Page

1. The Finite Difference Method for Various Time Steps (the flat plate)

2. The Finite Difference Method for Various Time Steps (the sphere)

3. Comparsion of Runge-Kutta and the Finite Difference Methods (the flat plate, $\Delta t=0.01$ )

4. Comparsion of Runge-Kutta and the Finite Difference Methods (the sphere, $\Delta t=0.01$ )

\section{Figure}

4.1 Graphical Representation for Crank-Nicolson Equation

5.1 Surface Temperature of a Flat Plate Cooled

by Convection and Radiation

(Runge-Kutta Method, $0.1 \leq t \leq 1.0$ )

5.2 Surface Temperature of a Flat Plate Cooled

by Convection and Radiation

(Runge-Kutta Method, $0.0001 \leq t \leq 0.01$ )

5.3 Surface Temperature of a Sphere Cooled

by Convection and Radiation

(Runge-Kutta Method, $0.001 \leq t \leq 0.1$ )

5.4 Surface Temperature of a Flat Plate Cooled

by Convection and Radiation

(Runge-Kutta Method of the First Order, $\Delta t=0.1,0.01$ ) 
5.5 Surface Temperature of a Flat Plate Cooled

by Convection and Radiation

(Finite Difference Method, $\Delta t=0.01,0.01 \leq t \leq 1.0$ )

5.6 Surface Temperature of a Sphere Cooled

by Convection and Radiation

(Finite Difference Method, $\Delta t=0.01,0.001$ )

5.7 Comparsion of Results for Cooling a Flat Plate $(\Delta t=0.01)$

5.8 Comparsion of Results for Cooling a Sphere $(\Delta \mathrm{t}=0.01)$

5.9 Surface Temperature of a Flat Plate Cooled

by Convection and Radiation

(Finite Difference Method, $\Delta t=0.01,1.0 \leq t \leq 10.0$ )

5.10 Surface Temperature of a Sphere Cooled

by Convection and Radiation

(Finite Difference Method, $\Delta t=0.01,1.0 \leq t \leq 10.0$ ) 


\section{CHAPTER I}

\section{ANALYTIC SOLUTIONS OF THE HEAT EQUATION SUBJECTED TO COMBINED CONVECTIVE AND RADIATIVE BOUNDARY CONDITIONS}

\section{1,1 Introduction}

During the 60's, space technology advanced so much that the research of the temperature behavior of bodies exposed to a deep space environment became crucial. In particular, transient heating or cooling of solids of different shapes by convection and thermal radiation was becoming highly important in many engineering applications. An example of these applications is the temperature distributions of rocket motors. An extensive investigation of the problem has been conducted and a lot of literature on the subject was published during the 60's and 70's. A detailed review of most of these papers is not intended here; instead a brief summary of the major ones will be given.

As early as 1962, Fairall, et.al. [6] generated a numericai solution for the problem using an explicit finite difference scheme; this paper served as a pioneer work in the area of the research. Later, various finite difference schemes were devised to deal with the nonlinear boundary condition. The main difficulty in these schemes is the appearance of severe oscillations in the determined temperature values for high heat flux situations. Von Rosenberg [10] proposed a hybrid of an iterative technique and implicit finite 
difference schemes to deal with the nonlinear boundary condition. On the other hand, Crosbie and Viskanta $[3,4]$ transformed the governing equations into a nonlinear Volterra integral equation of the second type and applied the method of successive approximations to solve the integral equation. Milton and Goss [8,9] developed some heuristic stability criteria for explicit finite difference schemes with nonlinear boundary conditions. It turns out that a very restrictive time step is required for numerical stability which may result in requiring a prohibitive amount of computer time to calculate the long time evolution of the solutions. Williams and Curry [12] surveyed several methods for treating the nonlinear boundary condition in implicit schemes and compared their accuracy and efficiency.

Nonlinearity is commonplace in natural phenomena. Unfortunately, a nonlinear problem often lends itself to a closed form solution. The problem of transient heat-conduction in a solid becomes nonlinear when the surface of the body is subjected to thermal radiation. When energy transfers through the wail of a body, two cases arise: convection and thermal radiation. The convective heat transfer describes the situation where heat is dissipated according to Newton's Law of cooling, which states that the rate at which heat is transferred from the body to a surrounding is proportional to the difference in temperature between the body and the environment. The boundary condition that describes convection is nonlinear except for the case where the heat-transfer coeffiecent is independent of surface temperature, which is technically called forced convection. The radiative heat transfer is based on the Stefan-Boltzmann Law, which states that the heat flux is 
proportional to the difference in temperature between the surface of power four and the source. Pure radiation or pure convection will occur whenever one mode of energy transfer predominates over the other.

It is the purpose of the thesis to consider the one-dimensional transient heat conduction problem resulting from a combined convective and radiative heat flux with the objective of determining the surface temperature fields using the numerical methods which are discussed in this study. Another purpose of this thesis is to explore the limitations and difficulties involved in these schemes. References to the work done in similar areas are presented to allow the reader further investigation.

Analytic solutions are derived in one dimension. However, the resulting solutions are in closed form, and thus impractical to use. Hence, numerical techniques will be studied and employed in the computer in an attempt to find an approximated solution. Numerical results, found by implementing some of the numerical methods discussed below will be presented and compared. In the conclusion, a numerical scheme is proposed as an alternative to the existing methods. It is open to the readers for justification.

Sections 1.3 and 1.4 describe the derivation of the integral representations of the one-dimensional transient heat conduction problem subjected to a combined convective and radiative boundary condition in a rectangular coordinate system. Two integral transform methods, namely the Laplace transform and the eigenvalue expansion, are presented. Observation 
and comparison are made for the integral equations to yield some useful information about the solutions.

In Chapters II and III, numerical methods for the solutions of nonlinear Volterra integral equations of the second type are described. In particular, the method of successive approximations and the Runge-Kutta method are outlined in detail. A brief remark is given for their advantages and limitations in finding solutions to the integral equation.

Chapter IV describes a numerical method which is directly applied to the governing partial differential equation. The technique is called the finite difference method. It is basically a hybrid of finite differencing techniques and an iterative scheme proposed. A suggestion is made for the improvement of the algorithm.

In Chapter V, numerical results produced by some of the discussed numerical schemes are presented. The implementation of various methods gave a practical sense of their advantages and limitations. Graphs and tables are set up in such a way that a comparison can be made.

In the next section, a statement of the problems is given. In the statement, the basic assumptions, the governing equation and the boundaryinitial conditions are included. 


\subsection{Statement of the Problem}

Considering the one-dimensional, transient, conduction heat transfer problem with combined convection and radiation at its surface, the following assumptions have been made:

1. One-dimensional heat transfer to a solid of a finite length.

2. The solid medium is pure, isotropic, homogeneous, and opaque to thermal radiation.

3. All thermodynamic and transport properties are independent of temperature.

4. The solid does not contain any heat sources or sinks.

5. The fluid is transparent to thermal radiation.

6. The fluid temperature and the ambient temperature are constant.

The non-dimensional form of the governing partial differential equation for the temperature $\mathrm{U}(\mathrm{x}, \mathrm{t})$ and the appropriate initial-boundary conditions are stated as follows:

$$
\frac{\partial^{2} U}{\partial x^{2}}=\frac{\partial U}{\partial t}, \quad 0<x<1, \quad \hat{\imath}>0
$$

Initiaí condition:

$$
\mathrm{U}(\mathrm{x}, 0)=\mathrm{g}(\mathrm{x}) \text {, }
$$

Boundary conditions:

$$
\begin{aligned}
& \text { (i) } \alpha_{1} \frac{\partial \mathrm{U}(0, \mathrm{t})}{\partial \mathrm{x}}-\alpha_{2} \mathrm{U}(0, \mathrm{t})=0 \\
& \text { (ii) } \frac{\partial \mathrm{U}(1, \mathrm{t})}{\partial \mathrm{x}}-\alpha_{3} \mathrm{U}(1, \mathrm{t})=-\mathrm{h} \mathrm{U}^{4}(1, \mathrm{t})
\end{aligned}
$$


where $\propto_{1}$ and $\alpha_{2}$ can be any real numbers, except both cannot be zero at the same time, $\propto_{3}$ is a non-zero real number, and $h$ is a positive real number.

Next section will consider partial differential equation (1.1) and initialboundary conditions $(1.2 \mathrm{a}, \mathrm{b}, \mathrm{c})$. The techniques for solving this problem will be the Laplace transform method and the eigenvalue expansion method. As an illustration, two special cases with specific values of $\propto_{1}, \propto_{2}, \propto_{3}$, and $h$ will be considered, and the analytic solutions of these cases at the surface will be derived. It will be shown that the surface temperature satisfies a singular Volterra integral equation of the second kind. At the end of the chapter, these solutions will be observed, and some useful information about the integral equations will be deduced.

\subsection{The Laplace Transform Method}

In this method, the Laplace transform of partial differential equation (1.1) and of the associated boundary conditions $(1.2 b, c)$ are first obtained with respect to time. The resulting boundary value problem is in terms of the Laplace transform of the required solution. Next, the equations are solved for the transformed temperature, and the solution of the stated problem can be found by taking the inverse Laplace transform of the transformed solution. As can be noticed, the Laplace inversion is of some difficulty. To simplify the situation, specific values of $\propto_{1}, \propto_{2}, \propto_{3}$, and $h$ are considered so that the inverse process is practical.

Now, define the transform of the temperature function, $U(x, t)$, with respect to time as follows: 


$$
f[U(x, t)](s)=\int_{0}^{\infty} U(x, t) e^{-s \imath} d t=\tilde{U}(x, s) .
$$

After transform, the temperature function is a function not only of $\mathrm{X}$ but also of the parameter S. Assuming that the derivatives with respect to $X$ pass through the transform, the followings are obtained:

$$
\begin{aligned}
& f\left[\frac{\partial U(x, t)}{\partial x}\right](s)=\int_{0}^{\infty} \frac{\partial U(x, t)}{\partial x} e^{-s t} d t=\frac{d \tilde{U}(x, s)}{d x} \\
& f\left[\frac{\partial^{2} U(x, t)}{\partial x^{2}}\right](s)=\int_{0}^{\infty} \frac{\partial^{2} U(x, t)}{\partial x^{2}} e^{-s t} d t=\frac{d^{2} \tilde{U}(x, s)}{d x^{2}} .
\end{aligned}
$$

The rule for transforming a derivative with respect to time can be found with integration by parts. Thus, the Laplace transform of the derivatives of $\mathrm{U}(\mathrm{x}, \mathrm{t})$ with respect to the transformed variable $\mathrm{t}$ is given by

$$
f\left[\frac{\partial U(x, t)}{\partial t}\right](s)=\int_{0}^{\infty} \frac{\partial U(x, t)}{\partial t} e^{-s t} d t=s \tilde{U}(x, s)-U(x, 0)
$$

Applying the Laplace transform to boundary-initial value problem (1.1), $(1.2 \mathrm{a}, \mathrm{b}, \mathrm{c})$, all time derivatives disappear, leaving the following ordinary differential equation in $\mathrm{X}$ and boundary conditions:

$$
\frac{d^{2} \tilde{U}(x, s)}{d x^{2}}-s \tilde{U}(x, s)=-g(x), \quad 0<x<1
$$

with

$$
\alpha_{1} \frac{d \tilde{U}(0, s)}{d x}-\alpha_{2} \tilde{U}(0, s)=0, \quad x=0,
$$




$$
\frac{d \tilde{U}(1, s)}{d x}-\alpha_{3} \tilde{U}(1, s)=-n E\left[U^{4}(1, s)\right], \quad x=1
$$

Notice that the initial condition, $\mathrm{g}(\mathrm{x})$, is incorporated in the ordinary differential equation. In order to solve $(1.7),(1.8 \mathrm{a}, \mathrm{b})$, the general solution of the corresponding homogeneous differential equation and a particular solution of (1.7) with $(1.8 \mathrm{a}, \mathrm{b})$ are needed to be found first. Now, consider the general solution of the homogeneous equation:

$$
\tilde{U}_{h}(x, s)=A e^{\lambda_{1} x}+B e^{\lambda_{2} x}
$$

where

$$
\lambda_{1,2}= \pm \sqrt{5}
$$

which are given by the roots of the auxiliary equation

$$
\left(\lambda^{2}-s\right)=0 \text {. }
$$

Next, variation of parameter method is used to solve a particular solution of (1.7). According to the method, the solution is first assumed to be the following form:

$$
\tilde{U}_{p}(x, s)=\tilde{U}_{1} v_{1}(x, s)+\tilde{U}_{2} v_{2}(x, s)
$$

where

$$
\tilde{U}_{1}(x, s) \text { and } \tilde{U}_{2}(x, s)
$$

are any two independent solutions of the correspondng homogeneous equation. In this case, choose

$$
\tilde{U}_{1}(x, s)=e^{\sqrt{s} x} \text { and } \tilde{U}_{2}(x, s)=e^{-\sqrt{s} x}
$$


The objective here is to find $v_{1}(x, S)$ and $v_{2}(x, s)$ such that the following equations are satisfied:

$$
\begin{aligned}
& e^{\sqrt{s} x} v_{1}^{\prime}(x, s)+e^{-\sqrt{s} x} v_{2}^{\prime}(x, s)=0 \\
& \sqrt{s} e^{\sqrt{s} x} v_{1}^{\prime}(x, s)-\sqrt{s} e^{-\sqrt{s} x} v_{2}^{\prime}(x, s)=-g(x)
\end{aligned}
$$

By Cramer's rule,

$$
v_{1}^{\prime}(x, s)=\frac{g(x) e^{-\sqrt{s} x}}{-2 \sqrt{s}}
$$

and

$$
v_{2}^{\prime}(x, s)=\frac{g(x) e^{\sqrt{s} x}}{2 \sqrt{s}}
$$

By integrating (1.15) and (1.16), they become

$$
\begin{aligned}
& v_{1}(x, s)-v_{1}(0, s)=-\int_{0}^{x} \frac{g\left(x^{\prime}\right) e^{-\sqrt{s} x^{\prime}} d x^{\prime}}{2 \sqrt{s}} \\
& v_{2}(x, s)-v_{2}(0, s)=\int_{0}^{x} \frac{g\left(x^{\prime}\right) e^{\sqrt{s} x^{\prime}} d x^{\prime}}{2 \sqrt{s}}
\end{aligned}
$$

Thus, the general solution to $(1.7),(1.8 \mathrm{a}, \mathrm{b})$ is given by

$$
\begin{aligned}
\tilde{U}(x, s) & =\tilde{U}_{h}(x, s)+\tilde{U}_{p}(x, s) \\
& =A e^{\sqrt{s} x}+B e^{-\sqrt{s} x}+e^{\sqrt{s} x} v_{1}(x, s)+e^{-\sqrt{s} x} v_{2}(x, s)
\end{aligned}
$$

where $A, B$ are constants and $v_{1}(x, S), v_{2}(x, S)$ are given by (1.17), (1.18), respectively.

To determine A and B, boundary conditions $(1.8 \mathrm{a}, \mathrm{b})$ are used. The derivative of $\mathrm{U}(\mathrm{x}, \mathrm{s})$ is found to be 


$$
\begin{gathered}
\frac{d \tilde{U}(x, s)}{d x}=A \sqrt{s} e^{\sqrt{s} x}-B \sqrt{s} e^{-\sqrt{s} x}+\sqrt{s} e^{\sqrt{s} x} v_{1}(x, s)+ \\
e^{\sqrt{s} x} v_{1}^{\prime}(x, s)+e^{-\sqrt{s} x} v_{2}^{\prime}(x, s)-\sqrt{s} e^{-\sqrt{s} x} v_{2}(x, s)
\end{gathered}
$$

When $x=0,(1.6 a),(1.18),(1.19)$, and the fact

$$
v_{1}^{\prime}(0, s)+v_{2}^{\prime}(0, s)=0
$$

give

$$
\begin{aligned}
& \alpha_{1}\left[A \sqrt{s}-B \sqrt{s}+\sqrt{s} v_{1}(0, s)-\sqrt{s} v_{2}(0, s)\right]- \\
& \alpha_{2}\left[A+B+v_{1}(0, s)+v_{2}(0, s)\right]=0 .
\end{aligned}
$$

Rearranging the terms, (1.20) becomes

$$
\begin{aligned}
& A\left(\alpha_{1} \sqrt{s}-\alpha_{2}\right)-B\left(\alpha_{1} \sqrt{s}+\alpha_{2}\right)= \\
& \left(\alpha_{1} \sqrt{s}+\alpha_{2}\right) v_{2}(0, s)-\left(\alpha_{1} \sqrt{s}-\alpha_{2}\right) v_{1}(0, s) .
\end{aligned}
$$

When $x=1,(1.8 \mathrm{~b}),(1.20),(1.21)$, and the fact

$$
e^{\sqrt{s}} v_{1}^{\prime}(1, s)+e^{-\sqrt{s}} v_{2}^{\prime}(1, s)=0
$$

give

$$
\begin{array}{r}
A \sqrt{s} e^{\sqrt{s}}-B \sqrt{s} e^{-\sqrt{s}}+\sqrt{s} e^{\sqrt{s}} v_{1}(1, s)-\sqrt{s} e^{-\sqrt{s}} v_{2}(1, s)- \\
\alpha_{3}\left[A e^{\sqrt{s}}+B e^{-\sqrt{s}}+e^{\sqrt{s}} v_{1}(1, s)+e^{-\sqrt{s}} v_{2}(1, s)\right]= \\
-h f\left[U^{4}(1, t)\right] .
\end{array}
$$

Again, manipulating the terms, (1.25) becomes

$$
\begin{aligned}
A\left(\sqrt{s} e^{\sqrt{s}}-\alpha_{3} e^{\sqrt{s}}\right)-B\left(\sqrt{s} e^{-\sqrt{s}}+\alpha_{3} e^{-\sqrt{s}}\right)= \\
\left(\sqrt{s} e^{-\sqrt{s}}+\alpha_{3} e^{-\sqrt{s}}\right) v_{2}(1, s)-\left(\sqrt{s} e^{\sqrt{s}}-\alpha_{3} e^{\sqrt{s}}\right) v_{1}(1, s)- \\
h f\left[U^{4}(1, t)\right] .
\end{aligned}
$$


Equations (1.23) and (1.26) form a system of two equations with two unknowns $A$ and $B$. By Cramer's rule, $A$ and $B$ are given by

$$
\begin{aligned}
A & =\frac{\left\{\left(\alpha_{1} \sqrt{s}-\alpha_{2}\right) v_{1}(0, s)+\left\{\alpha_{1} \sqrt{s}+\alpha_{2}\right)\left[v_{2}(1, s)-v_{2}(0, s)\right]\right\}\left(\sqrt{s} e^{-\sqrt{8}}+\alpha_{3} e^{-\sqrt{8}}\right)}{\left(\alpha_{2}-\alpha_{3} \alpha_{1}\right) \sqrt{s}\left(\varepsilon^{\sqrt{8}}+e^{-\sqrt{8}}\right)+\left(\alpha_{1} s-\alpha_{3} \alpha_{2}\right)\left(e^{\sqrt{8}}-e^{-\sqrt{8}}\right)} \\
& +\frac{-\left[\left(\alpha_{1} \sqrt{s}+\alpha_{2}\right) v_{1}(1, s)\right]\left(\sqrt{s} e^{\sqrt{8}}-\alpha_{3} e^{\sqrt{8}}\right)-h z\left[U^{4}(1, t)\right]\left(\alpha_{1} \sqrt{s}+\alpha_{2}\right)}{\left(\alpha_{2}-\alpha_{3} \alpha_{1}\right) \sqrt{s}\left(e^{\sqrt{8}}+e^{-\sqrt{8}}\right)+\left(\alpha_{1} s-\alpha_{3} \alpha_{2}\right)\left(e^{\sqrt{8}}-e^{-\sqrt{8}}\right)}
\end{aligned}
$$

$$
\begin{aligned}
B & =\frac{\left\{\left(\alpha_{1} \sqrt{s}-\alpha_{2}\right)\left[v_{1}(0, s)-v_{1}(1, s)\right]-\left(\alpha_{1} \sqrt{s}+\alpha_{2}\right) v_{2}(0, s)\right\}\left(\sqrt{s} e^{\sqrt{8}}-\alpha_{3} e^{\sqrt{8}}\right)}{\left(\alpha_{2}-\alpha_{3} \alpha_{1}\right) \sqrt{s}\left(e^{\sqrt{8}}+e^{-\sqrt{8}}\right)+\left(\alpha_{1} s-\alpha_{3} \alpha_{2}\right)\left(e^{\sqrt{8}}-e^{-\sqrt{8}}\right)} \\
& +\frac{-h f\left[U^{4}(1, t)\right]\left(\alpha_{1} \sqrt{s}-\alpha_{2}\right)+\left[\left(\alpha_{1} \sqrt{s}-\alpha_{2}\right) v_{2}(1, s)\right]\left(\sqrt{s} e^{-\sqrt{8}}+\alpha_{3} e^{-\sqrt{8}}\right)}{\left(\alpha_{2}-\alpha_{3} \alpha_{1}\right) \sqrt{s}\left(e^{\sqrt{8}}+e^{-\sqrt{8}}\right)+\left(\alpha_{1} s-\alpha_{3} \alpha_{2}\right)\left(e^{\sqrt{8}}-e^{-\sqrt{8}}\right)}
\end{aligned}
$$

Thus, the general solution of $(1.7),(1.8 \mathrm{a}, \mathrm{b})$ is given by (1.20) where A, $B, v_{1}(x, S)$, and $v_{2}(X, S)$ are (1.27), (1.28), (1.17), and (1.18), respectively. Theoretically, the analytic solution of partial differential equation (1.1) with initial-boundary conditions $(1.2 a, b, c)$ can be oblained by taking the Laplace inversion of $\tilde{U}(x, s)$, and thus, the surface solution can be found by putting $x$ equal to one in $U(x, S)$. In practice, however, the inverse Laplace process is highly unstable and difficult. In the next section, two special cases will be considered so that the inversion is possible. In each case, values for the parameters correspond to a specific geometrical configuration of a body. 
CASE 1: $\propto_{1}=1, \alpha_{2}=0, \alpha_{3}=-1, h=1$

This set of values corresponds to the case where a solid slab of length 1 , usually called a "flat plate," with an initial temperature is being heated or cooled by combined convection and radiation. If the given values for the parameters are substituted in (1.20), the transformed surface temperature is given by

$$
\tilde{U}(1, s)=A e^{\sqrt{s}}+B e^{-\sqrt{s}}+e^{\sqrt{s}} v_{1}(1, s)+e^{-\sqrt{s}} v_{2}(1, s)
$$

where

$$
\begin{aligned}
A & =\frac{\left[\sqrt{s} v_{1}(0, s)+\sqrt{s v_{2}}(1, s)-\sqrt{s v_{2}}(0, s)\right]\left(\sqrt{s} e^{-\sqrt{8}}-e^{-\sqrt{8}}\right)}{\sqrt{s}\left(\varepsilon^{\sqrt{8}}+e^{-\sqrt{8}}\right)+s\left(e^{\sqrt{8}}-e^{-\sqrt{8}}\right)} \\
& +\frac{-\left[\sqrt{s} v_{1}(1, s)\right]\left(\sqrt{s} e^{\sqrt{8}}+e^{\sqrt{8}}\right)-h f\left[U^{4}(1, t)\right] \sqrt{s}}{\sqrt{s}\left(e^{\sqrt{8}}+e^{-\sqrt{8}}\right)+s\left(e^{\sqrt{8}}-e^{-\sqrt{8}}\right)} \\
B & =\frac{\left[\sqrt{s} v_{1}(0, s)-\sqrt{s v_{1}}(1, s)-\sqrt{s v_{2}}(0, s)\right]\left(\sqrt{s} e^{\sqrt{8}}+e^{\sqrt{8}}\right)}{\left(\alpha_{2}-\alpha_{3} \alpha_{1}\right) \sqrt{s}\left(e^{\sqrt{8}}+e^{-\sqrt{8}}\right)+\left(\alpha_{1} s-\alpha_{3} \alpha_{2}\right)\left(e^{\sqrt{8}}-e^{-\sqrt{8}}\right)} \\
& +\frac{-h \varepsilon\left[U^{4}(1, t)\right] \sqrt{s}+\left[\sqrt{s} v_{2}(1, s)\right]\left(\sqrt{s} e^{-\sqrt{8}}-e^{-\sqrt{8}}\right)}{\left(\alpha_{2}-\alpha_{3} \alpha_{1}\right) \sqrt{s}\left(e^{\sqrt{8}}+e^{-\sqrt{8}}\right)+\left(\alpha_{1} s-\alpha_{3} \alpha_{2}\right)\left(e^{\sqrt{8}}-e^{-\sqrt{8}}\right),} \\
v_{1}(1, s)=-\int_{0}^{1} \frac{g\left(x^{\prime}\right) e^{-\sqrt{s} x^{\prime}} d x^{\prime}}{2 \sqrt{s}}+v_{1}(0, s) &
\end{aligned}
$$

and

$$
v_{2}(1, s)=\int_{0}^{1} \frac{g\left(x^{\prime}\right) e^{\sqrt{s} x^{\prime}} d x^{\prime}}{2 \sqrt{s}}+v_{2}(0, s)
$$

Substituting (1.30)-(1.33) in (1.29) and simplifying the result gives 


$$
\begin{aligned}
\tilde{U}(1, s) & =\frac{\left(\int_{0}^{1} g\left(x^{\prime}\right) e^{\sqrt{s} x^{\prime}} d x^{\prime}+\int_{0}^{1} g\left(x^{\prime}\right) e^{-\sqrt{s} x^{\prime}} d x^{\prime}\right)}{\left(e^{\sqrt{s}}+e^{-\sqrt{s}}\right)+\sqrt{s}\left(e^{\sqrt{s}}-e^{-\sqrt{s}}\right)} \\
& -\frac{h £\left[U^{4}(1, t)\right]\left(e^{\sqrt{s}}+e^{-\sqrt{s}}\right)}{\left(e^{\sqrt{s}}+e^{-\sqrt{s}}\right)+\sqrt{s}\left(e^{\sqrt{s}}-e^{-\sqrt{s}}\right)} .
\end{aligned}
$$

Suppose the initial temperature is chosen to be 1 , that is,

$$
g(x)=1
$$

Boundary conditions associated with the given values of the parameters and initial condition (1.35) constitute a cooling process. With (1.35), the transformed surface temperature becomes

$$
\begin{aligned}
\tilde{U}(1, s) & =\frac{\frac{1}{\sqrt{s}}\left(e^{\sqrt{s}}-e^{-\sqrt{s}}\right)}{\left(e^{\sqrt{s}}+e^{-\sqrt{s}}\right)+\sqrt{s}\left(e^{\sqrt{s}}-e^{-\sqrt{s}}\right)} \\
& -\frac{h E\left[U^{4}(1, t)\right]\left(e^{\sqrt{s}}+e^{-\sqrt{s}}\right)}{\left(e^{\sqrt{s}}+e^{-\sqrt{s}}\right)+\sqrt{s}\left(e^{\sqrt{s}}-e^{-\sqrt{s}}\right)} .
\end{aligned}
$$

If (1.36) is multiplied throughout by

$$
\frac{1}{\sqrt{s}}\left[\frac{\left(e^{\sqrt{s}}+e^{-\sqrt{s}}\right)+\sqrt{s}\left(e^{\sqrt{s}}-e^{-\sqrt{s}}\right)}{\left(e^{\sqrt{s}}-e^{-\sqrt{s}}\right)}\right]
$$

and then simplified,

$$
\tilde{U}(1, s)=\frac{1}{s}-\frac{f\left[h U^{4}(1, t)+U(1, t)\right]\left(e^{\sqrt{s}}+e^{-\sqrt{s}}\right)}{\sqrt{s}\left(e^{\sqrt{s}}-e^{-\sqrt{s}}\right)}
$$

is obtained. (1.37) is ready to be inverted. In order to perform the Laplace inversion of (1.37), two inverse Laplace transforms, namely, 


$$
f^{-1}\left[\frac{1}{s}\right] \text { and } f^{-1}\left[\frac{\left(e^{\sqrt{s}}+e^{-\sqrt{s}}\right)}{\sqrt{s}\left(e^{\sqrt{s}}-e^{-\sqrt{s}}\right)}\right]
$$

have to be computed. In fact, they can be found from any standard Laplace transform table [13]. By the convolution theorem, the surface temperature in time $t$ is given by

$$
U(1, \hat{\tau})=1-\int_{0}^{t} \frac{\left[1+2 \sum_{k=1}^{\infty} e^{\frac{-k^{2}}{(t-\tau)}}\right]}{\sqrt{\pi(t-\tau)}}\left[U^{4}(1, \tau)+U(1, \tau)\right] d \tau
$$

and by the poisson summation formula [14], (1.38) can be rewritten as

$U(1, t)=1-\int_{0}^{\tau}\left[1+2 \sum_{k=1}^{\infty} e^{-k^{2} \pi^{2}(t-\tau)}\right]\left[U^{4}(1, \tau)+U(1, \tau)\right] d \tau$

Hence, the problem of transient cooling of a flat plate by combined convection and thermal radiation has been reduced to solving a nonlinear Volterra integral equation of the second kind.

CASE 2: $\alpha_{1}=0, \alpha_{2}=-1, \alpha_{3}=1, h=1$.

This set of values correspond to the case where a spherical body of radius 1 with an initial uniform temperature is being heated or cooled by combined convection and radiation. Since the procedures used to solve the problem are basically those described in case 1 , the mathematical details will be omitted and only the main steps will be presented. 
Consider equation (1.20), the general solution of the boundary value problem. The given values for the parameters are first substituted in (1.17), (1.18), (1.27), and (1.28). Then, (1.20) is simplified as in the previous case. After a tedious calculation, the transformed surface temperature is given by

$$
\begin{aligned}
\tilde{U}(1, s) & =\frac{\left(\int_{0}^{1} g\left(x^{\prime}\right) e^{-\sqrt{s} x^{\prime}} d x^{\prime}-\int_{0}^{1} g\left(x^{\prime}\right) e^{\sqrt{s} x^{\prime}} d x^{\prime}\right)}{\left(e^{\sqrt{s}}-e^{-\sqrt{s}}\right)-\sqrt{s}\left(e^{\sqrt{s}}+e^{-\sqrt{s}}\right)} \\
& +\frac{h \varepsilon\left[U^{4}(1, t)\right]\left(e^{\sqrt{s}}-e^{-\sqrt{s}}\right)}{\left(e^{\sqrt{s}}-e^{-\sqrt{s}}\right)-\sqrt{s}\left(e^{\sqrt{s}}+e^{-\sqrt{s}}\right)}
\end{aligned}
$$

Suppose the initial temperature is chosen to be

$$
g(x)=x
$$

Boundary conditions associated with the given values of the parameters and initial conditon (1.41) constitute a cooling process. With (1.41), the transformed surface temperature becomes

$$
\tilde{U}(1, s)=\frac{1}{s}-\frac{h f\left[U^{4}(1, t)\right]\left(e^{\sqrt{s}}-e^{-\sqrt{s}}\right)}{\sqrt{s}\left(e^{\sqrt{s}}+e^{-\sqrt{s}}\right)-\left(e^{\sqrt{s}}-e^{-\sqrt{s}}\right)}
$$

which is ready to be inverted. In order to perform the Laplace inversion of (1.42), two inverse Laplace transforms, namely,

$$
f^{-1}\left[\frac{1}{s}\right] \text { and } E^{-1}\left[\frac{\left(e^{\sqrt{s}}-e^{-\sqrt{s}}\right)}{\sqrt{s}\left(e^{\sqrt{s}}+e^{-\sqrt{s}}\right)-\left(e^{\sqrt{s}}-e^{-\sqrt{s}}\right)}\right]
$$


have to be computed. The first inverse Laplace transform is obvious. However, the second one is not trivial. Details of the derivation of the second inverse Laplace transform are available in [1]. The surface temperature in time $t$, obtained by inverting (1.42), is

$$
U(1, t)=1-\int_{0}^{t}\left[3+2 \sum_{k=1}^{\infty} e^{-\beta_{x}^{2}(t-\tau)}\right] U^{4}(1, \tau) d \tau
$$

where $\beta_{\mathrm{K}}$ is the $\mathrm{k}$-th positive root of the transcendental equation

$$
\beta_{k}=\tan \beta_{k}
$$

Hence, the problem of transient cooling of a sphere by combined convection and thermal radiation has been again reduced to solving a nonlinear Volterra integral equation of the second kind.

As experienced, one of the drawbacks of the Laplace transform method is that there are only a few cases in which the transformed solution can be practically inverted into the required solution. In the next section, the eigenvalue expansion method is introduced as an alternative to the above method. One may find that the eigenvalue method is more practical for solving the analytic solution of the heat equation with nonlinear boundary conditions. 


\subsection{The Eigenvalue Expansion Method}

The fundamental idea of the eigenvalue expansion method is to transform the given boundary value problem by the eigenfunctions obtained from the associated eigenvalue problem. By the completeness theorem and by the orthogonal property of the eigenfunctions, the solution of the problem can be expressed as an infinite sum of the eigenfunctions with appropiate coefficients. Applying these procedures to partial differential equation (1.1) and initial-boundary conditions $(1.2 \mathrm{a}, \mathrm{b}, \mathrm{c})$ yields the following main results:

First, solve for the associated eigenvalue problem:

$$
\frac{d^{2} X(x)}{d x^{2}}+\beta^{2} X(x)=0, \quad 0<x<1
$$

Boundary conditions:

$$
\alpha_{1} \frac{d X(0)}{d x}-\alpha_{2} X(0)=0
$$

and

$$
\frac{d X(1)}{d x}-a_{3} X(1)=0
$$

Parameters $\propto_{1}$ and $\propto_{2}$ can be any real numbers except they cannot be zero at the same time; whereas, $\alpha_{3}$ is a non-zero real number. According to the theorem of ordinary differential equations, the general solution of (1.45) is

$$
X(x)=c_{1} \cos (\beta x)+c_{2} \sin (\beta x)
$$

Applying boundary conditions (1.46) and (1.47) to equation (1.48) gives the following system of equations: 


$$
\begin{aligned}
& \alpha_{1} \beta c_{2}=\alpha_{2} c_{1}, \\
& \left(c_{2} \beta-c_{1} \alpha_{3}\right) \cos \beta=\left(c_{1} \beta+c_{2} \alpha_{3}\right) \sin \beta .
\end{aligned}
$$

Note that boundary value problem $(1.45-1.47)$ is in the class of SturmLiouville problems for which all eigenvalues are real and the eigenfunctions corresponding to arbitary eigenvalues are orthogonal. Thus, if the parameters in (1.49) and (1.50) are specified, there will exist eigenvalues, $\beta_{n}$, and the corresponding eigenfunctions, $X_{n}(x)$, such that the temperature function, $U(X, t)$, can be expanded in Fourier expansion of the form

$$
U(x, t)=\sum_{n=1}^{\infty} U_{n}(t) X_{n}(x),
$$

where the Fourier coefficients, $U_{n}(t)$, are given by

$$
U_{n}(t)=\int_{0}^{1} U(x, t) X_{n}(x) d x
$$

Now, taking the finite Fourier integral transform of heat equation (1.1) with respect to $X_{n}(X)$ gives

$$
\frac{d}{d t} \int_{0}^{1} U(x, t) X_{n}(x) d x=\int_{0}^{1} \frac{\partial^{2} U}{\partial x^{2}} X_{n}(x) d x
$$

Performing parts integration of the right-hand expression in equation (1.53) yields the following ordinary differential equation for $U_{n}(t)$

$$
\begin{aligned}
\frac{d U_{n}(t)}{d t} & =\frac{\partial U(1, t)}{\partial x} X_{n}(1)-\frac{\partial U(0, t)}{\partial x} X_{n}(0)-U(1, t) X_{n}^{\prime}(1) \\
& +U(0, t) X_{n}^{\prime}(0)+\int_{0}^{1} U(x, t) X_{n}^{\prime}(x) d x
\end{aligned}
$$


With boundary conditions (1.46) and (1.47), the right hand side of (1.54) can be simplified. Then, by the integrating factor method, the solution of equation (1.54) can be obtained. Hence, the resulting integral equation for $\mathrm{U}(\mathrm{x}, \mathrm{t})$ takes the form of (1.51) with $\mathrm{U}_{\mathrm{n}}(\mathrm{t})$ solved in (1.54). Lastly, by putting $X=1$, a nonlinear Volterra integral equation of the second kind for the surface temperature $U(1, t)$ is obtained.

As in the previous section, the integral equation for the surface temperature will be explicitly determined for two special cases: the flat plane and the sphere. Details of the derivation of the solution will be produced in the case of flat plane, but only major results will be given in the case of sphere.

CASE 1: $\alpha_{1}=1, \alpha_{2}=0, \alpha_{3}=-1, h=1$

As mentioned in Section 1.3, this set of parameters corresponds to the geometrical configuration of a flat plate. Substituting the values of $\propto_{1}, \propto_{2}$, and $\alpha_{3}$ in (1.49) and (1.50), $C_{1}$ equals zero, and (1.50) leads to

$$
\begin{aligned}
\cos \beta_{n} & =\beta_{n} \sin \beta_{n} \\
\Rightarrow \quad & \frac{1}{\beta_{n}}=\tan \beta_{n} .
\end{aligned}
$$

So, the family of orthogonal eigenfunctions are

$$
X_{n}(x)=\cos \left(\beta_{n} x\right)
$$

where $n=1,2,3, \ldots$, and $\beta_{n}$ is the set of distinct eigenvalues such that they are the roots of (1.55) with the property

$$
0<\beta_{1}<\beta_{2}<\beta_{3}<\cdots \text {. }
$$


Next, applying the finite Fourier integral transform of heat equation (1.1) with respect to $X_{n}(X)$, yields (1.54). Using boundary conditions

$$
\begin{aligned}
& \frac{\partial U(0, t)}{\partial x}=0 \\
& \frac{\partial U(1, t)}{\partial x}+U(1, t)=-n U^{4}(1, t) \\
& \frac{d X(1)}{d x}+X(1)=0 \\
& \frac{d X(0)}{d x}=0
\end{aligned}
$$

and the fact that

$$
X_{n}^{\prime \prime}(x)=-\beta_{n}^{2} X_{n}(x)
$$

produces the following ordinary differential equation for $U_{n}(t)$

$$
\frac{d U_{n}(t)}{d t}+\beta_{n}^{2} U_{n}(t)=-n X_{n}(1) U^{4}(1, t)
$$

By the technique for solving a first order differential equation, the solution of equation (1.62) is found to be

$$
U_{n}(t)=U_{n}(O) e^{-\beta_{n}^{2} t}-h X_{n}(1) \int_{0}^{t} e^{-\beta_{n}^{2}(t-\tau)} U^{4}(1, \tau) d \tau
$$

where $U_{n}(O)$ is defined as

$$
\int_{0}^{1} g(x) X_{n}(x) d x
$$

Thus, with $h=1$, the integral equation for $U(x, t)$ takes the form 


$$
U(x, t)=\sum_{n=1}^{\infty}\left\{\frac{\left(U_{n}(0) e^{-\beta_{x}^{2} \tau}-n X_{n}(1) \int_{0}^{\uparrow} e^{-\beta_{x}^{2}(t-\tau)} U^{4}(1, \tau) d \tau\right)}{\int_{0}^{1} X_{n}^{2}(x) d x} X_{n}(x)\right\}
$$

where $U_{n}(O)$ and $X_{n}(X)$ are defined by (1.64) and (1.56), respectively.

Lastly, by putting $X=1$, the integral equation for the surface temperature $\mathrm{U}(1, \mathrm{t})$ is determined to be

$$
\begin{aligned}
& U(1, t)=\sum_{n=1}^{\infty}\left(e^{-\beta_{n}^{2} t} X_{n}(1) \frac{\int_{0}^{1} g(x) X_{n}(x) d x}{\int_{0}^{1} X_{n}^{2}(x) d x}\right) \\
&-\int_{0}^{t}\left(\sum_{n=1}^{\infty} \frac{X_{n}^{2}(1) e^{-\beta_{n}^{2}(t-\tau)}}{\int_{0}^{1} X_{n}^{2}(x) d x}\right) U^{4}(1, \tau) d \tau,
\end{aligned}
$$

where $g(x)$ is the initial condition, $X_{n}(x)$ and $\beta_{n}$ are defined as above.

CASE 2: $\alpha_{1}=0, \alpha_{2}=-1, \alpha_{3}=1, h=1$.

In this case, a spherical body is considered. In a similar fashion, the family of orthogonal eigenfunctions can be found and are given by

$$
X_{n}(x)=\sin \left(\beta_{n} x\right)
$$

where $\mathbf{n}=1,2,3, \ldots$, and $\beta_{n}$ is the set of distinct eigenvalues such that they are the roots of

$$
\beta_{n}=\tan \beta_{n}
$$


with the property

$$
0<\beta_{1}<\beta_{2}<\beta_{3}<\cdots .
$$

After applying the finite Fourier integral transform of heat equation (1.1) with respect to $X_{n}(X)$, the following ordinary differential equation for $U_{n}(t)$ is obtained:

$$
\frac{d U_{n}(t)}{d t}+\beta_{n}^{2} U_{n}(t)=-h X_{n}(1) U^{4}(1, t)
$$

Thus, the solution of equation (1.68) is

$$
U_{n}(t)=U_{n}(0) e^{-\beta_{n}^{2} \tau}-n X_{n}(1) \int_{0}^{t} e^{-\beta_{n}^{2}(t-\tau)} U(1, \tau)^{4} d \tau
$$

where $U_{n}(O)$ is defined as

$$
\int_{0}^{1} g(x) X_{n}(x) d x
$$

Thus, with $h=1$, the integral equation for $U(x, t)$ takes the form

$$
U(x, t)=\sum_{n=1}^{\infty}\left\{\frac{\left(U_{n}(0) e^{-\beta_{n}^{2} t}-n X_{n}(1) \int_{0}^{t} e^{-\beta_{n}^{2}(t-\tau)} U^{4}(1, \tau) d \tau\right)}{\int_{0}^{1} X_{n}^{2}(x) d x} X_{n}(x)\right\}
$$


where $U_{n}(O)$ and $X_{n}(X)$ are defined by (1.70) and (1.66), respectively. Lastly, by putting $X=1$, the integral equation for the surface temperature $U(1, t)$ becomes

$$
\begin{aligned}
U(1, t)= & \sum_{n=1}^{\infty}\left(e^{-\beta_{n}^{2} t} X_{n}(1) \frac{\int_{0}^{1} g(x) X_{n}(x) d x}{\int_{0}^{1} X_{n}^{2}(x) d x}\right) \\
& -\int_{0}^{t}\left(\sum_{n=1}^{\infty} \frac{X_{n}^{2}(1) e^{-\beta_{n}^{2}(t-\tau)}}{\int_{0}^{1} X_{n}^{2}(x) d x}\right)^{4}(1, \tau) d \tau,
\end{aligned}
$$

where $g(x)$ is the initial condition, $X_{n}(x)$ and $\beta_{n}$ are defined as above. 


\subsection{Remarks}

The solution presented above is not complete in the sense that the surface temperature is only determined for two cases, namely, the flat plate and the sphere. The solutions for other geometrical configurations can be found in some of the literature listed in the reference section $[1,4]$.

All of the surface temperature solutions which have been derived in each case by both methods fall into the form

$$
U(1, t)=\Phi(t)-h \int_{0}^{t}\left(a+\sum_{k=1}^{\infty} b_{k} e^{-c_{k}^{2}(t-\tau)}\right) F[U(1, \tau)] d \tau
$$

where $F$ is a nonlinear function of $U(1, t)$, and $C_{k}, b_{k}, a$, and $h$ are some constants. Equation (1.72) is a nonlinear Volterra integral equation of the second kind. $\Phi(t)$ is a function which is usually called the "lag" part of the integral equation; the intergal in the second term of (1.72) is often referred as the "Volterra" part of the integral equation. In addition, the integrand of the Volterra part is called the "kernel" of the integral equation. As these integral equations are being examined, several "facts" about (1.72) are concluded and summarized as follows:

1. All of these integral equations are singular beacuse as $\tau$ approaches $t$, the kernels blow up to infinity.

2. All the infinite series satisfy the following property:

If $f(t-\tau)$ is used to denote an infinite series, then $\lim _{t \rightarrow \infty} f(t)=$ constant.

3. The lag part, $\Phi(t)$, and the kernel of the integral equations are determined by the geometry of the body considered. 
An interesting fact which can be observed when (1.65) and (1.71) are compared with (1.39) and (1.43) is that the lag part of the integral equation derived by the eigenvalue expansion method diverges when time is equal to zero. Theoretically, this situation should not happen; thus, it creates a subtlety that cannot be explained.

The above "facts" are concluded from only two special cases with specific choices of the initial condition and are thus open to the reader for justification of their validity in a more general context. In the next three chapters, three different numerical methods for solving the problem stated in Section 1.2 will be introduced. Both the method of successive approximations and the Runge-Kutta method are numerical techniques used to deal with the integral representation of the problem, whereas the finite difference method is directly applied to the governing equations. 


\section{CHAPTER II}

\section{THE METHOD OF SUCCESSIVE APPROXIMATIONS}

\subsection{Introduction}

The surface temperature of a body subjected to a combined convective and radiative boundary condition, as seen in Chapter 1 , is given by the solution of a singular nonlinear Volterra integral equation of the second kind. Since the integral equation is in closed form, numerical techniques seem to be the most practical way to tackle the problem. Over the past twenty years, a lot of research has been done, mostly by Russians, on the numerical solution of an integral equation of the form

$$
U(1, t)=\Phi(t)-h \int_{0}^{t}\left(a+\sum_{k=1}^{\infty} b_{k} e^{-c_{k}^{2}(t-\tau)}\right) F[U(1, \tau)] d \tau
$$

Among the existing numerical methods for solving (2.1), the method of successive approximations is the most popular one. It is based on the idea that the set of successive functions defined by

$$
y_{n+1}(t)=\Phi(t)-h \int_{0}^{t} k(t-\tau) F\left(y_{n}(\tau)\right) d \tau
$$

converges to a solution of (2.1) in every finite interval of time [1]. In the following section, the method of solution will be outlined, and at the end of the chapter, comment will be made on the technique. 


\subsection{Outline of the Method}

Consider the time domain on which integral equation (2.1) is to be solved. Suppose the domain is partitioned into $\mathrm{N}$ intervals. For the first time interval, $0 \leq t \leq t_{1}$, the approximated solution of the integral equation can be obtained by using the iteration procedure of

$$
U_{n+1}(1, t)=\Phi(t)-h \int_{0}^{t} k(t-\tau) F\left(U_{n}(1, \tau)\right) d \tau
$$

until the error between the last two approximations is less than a predefined small number. Next, consider the second time interval, $t_{1} \leq t \leq t_{2}$. In this interval,

$$
U_{n+1}(1, t)=\Phi(t)-h \int_{0}^{t} k(t-\tau) F\left(U_{n}(1, \tau)\right) d \tau
$$

can be broken into

$$
\begin{aligned}
U_{n+1}(1, t) & =\Phi(t)-h \int_{0}^{t_{1}} k(t-\tau) F(U(1, \tau)) d \tau \\
& -h \int_{t_{1}}^{t} k(t-\tau) F\left(U_{n}(1, \tau)\right) d \tau .
\end{aligned}
$$

Since $U(1, t)$ is determined for $0 \leq t \leq t_{1}$, the first integral,

$$
n \int_{0}^{i_{1}} k(t-\tau) F(U(1, \tau)) d \tau \text {, }
$$

is known, and thus, the iteration procedure is only needed for the second integral. In general, for the $i$-th time interval, the iteration procedure is given by 


$$
\begin{aligned}
U_{n+1}(1, t) & =\Phi(t)-n \int_{0}^{\tau_{i-1}} k(t-\tau) F(U(1, \tau)) d \tau \\
& -n \int_{i_{i-1}}^{\tau} k(t-\tau) F\left(U_{n}(1, \tau)\right) d \tau,
\end{aligned}
$$

where $t_{i-1} \leq t \leq t_{i}$.

As the procedure continues along the time scale, the surface temperature is known for all desired time. One may notice that as the algorithm is carried out, the singularity of the Volterra part of the integral equation creates difficulties when the integral part is evaluated numerically. In order to handle the difficulty appropriately, one has to know the nature of the singularity which the kernel possesses. To illustrate the idea, consider the integral of the following form:

$$
\int_{a}^{b} \frac{f(z)}{\sqrt{b-z}} d z
$$

This integral is often found in the integral representation of the stated problem. Integral (2.7) posesses a singularity which can be removed by the use of the transformation

$$
z=a+(b-a)\left(1-x^{2}\right)
$$

Then, by using a suitable Gaussian Quadrature formula, the integral can be evaluated accurately. Normally, one usually comes up with an integral with a stronger singularity.

The iteration procedure outlined above needs a starting value. Generally, the algorithm will converge faster to the exact solution if the starting value is close to the exact solution. Thus, choosing the initial approximation is a very crucial procedure. Based on the fact that the solution 
of the stated problem is continuous, one can choose the temperature at the previous time level as the first approximation of the method when a small time step is used.

The method of successive approximations has been applied to solve integral equation (2.1). In particular, a method used to tackle a simple type of singularity, which one may encounter when evaluating the Volterra part numerically, has been discussed. Since numerical integration is one of the key steps in the method, the choice of the numerical integration scheme does affect the overall performance of the algorithm. One can improve the accuracy of the successive approximations method by appropriately choosing a numerical quadrature that can best deal with the singularity found in the integral equation. Although the procedure outlined above may seem simple, it has been shown that the method is impractical for large times [3]. 


\section{CHAPTER III}

\section{THE RUNGE-KUTTA METHOD}

\subsection{Introduction}

This chapter considers another way to deal with the integral equation representing the stated heat conduction problem. The method is called the Runge-Kutta method which was first introduced by Crosbie and Viskanta [5] in 1968. The basic idea of the method is based on an approximation of the kernel by a separable kernel. The integral equation is differentiated with respect to time and transformed into a nonlinear differential equation. Using the Runge-Kutta method, a well-known numerical scheme for solutions of ordinary differential equations, the surface temperature at a desired time is determined. The order of approximation of the method is determined by the order of the ordinary differential equation. What is so different about this method versus the other numerical schemes is that, instead of solving an integral equation directly, the Volterra integral equation is first reduced to a system of nonlinear ordinary differential equations and then soived numerically. Notice that the method is not exact in a sense that the approximation of the kernel will not be practical if times are small. In general, the accuracy of the approximation of the kernel increases with time and with order. In the next section, the method will be outlined in detail as it is applied to integral equation (1.72). In addition, as an example, the formulas 
for the third and the fifth order approximation of the method will be produced explicitly.

\subsection{Outline of the method}

Consider the integral equations derived in chapter 1. Generally, the integral representation for the dimensionless surface temperature, $U(1, t)$, of the body that we have considered can be written as

$$
U(1, t)=\Phi(t)-\int_{0}^{t} k(t-\tau) F(U(1, \tau)) d \tau
$$

where

$$
k(t-\tau)=\beta_{o}+\sum_{k=1}^{\infty} \beta_{k} e^{-\alpha_{k}^{2}(t-\tau)}
$$

The function $F(U(1, t))$ is the surface heat flux; whereas, $\alpha$ 's and $\beta$ 's are eigenvalues and coefficients, respectively. According to chapter 1 , the infinite series, $k(t-\tau)$, satisfies the following property:

$$
\lim _{t \rightarrow \infty} k(t)=\beta_{0}
$$

This condition becomes a necessary condition for an iñtegral equation io which the method is applied. Now, assume $\Phi(t)$ is a bounded differentiable function. The Nth-order approximation of $k(t-\tau)$ is giving by taking the first $\mathrm{N}$ terms of the infinite summation. So, (3.2) becomes

$$
k(t-\tau)=\beta_{0}+\sum_{k=1}^{N} \beta_{k} e^{-\alpha_{k}^{2}(t-\tau)} \text {. }
$$

To differentiate (3.1) with $(3.4)$, let 


$$
I_{k}(t)=e^{-\alpha_{k}^{2} t} \int_{0}^{T} e^{\alpha_{k}^{2} \tau} F(U(1, \tau)) d \tau \text {. }
$$

Therefore, $\mathrm{U}(1, \mathrm{t})$ can be rewritten as

$$
U(1, t)=\Phi(t)-\beta_{0} \int_{0}^{i} F(U(1, \tau)) d \tau-\sum_{k=1}^{N} \beta_{k} I_{k}(t)
$$

Differentiating with respect to time, equation (3.6) becomes

$$
\begin{aligned}
U^{(1)}(1, t) & =\Phi^{(1)}(t)-\beta_{o} F(U(1, t)) \\
& -\sum_{k=1}^{N} \beta_{k}\left[F(U(1, t))-\alpha_{k}^{2} I_{k}(t)\right]
\end{aligned}
$$

If one keeps on differentiating (3.6), the first $m$ derivatives of (3.6) are given by the following formulas:

$$
\begin{aligned}
U^{(1)}(1, t)= & \Phi^{(1)}(t)-\beta_{o} F(U(1, t), t) \\
& -\sum_{k=1}^{N} \beta_{k}\left[F(U(1, t))-\alpha_{k}^{2} I_{k}(t)\right] \\
U^{(2)}(1, t)= & \Phi^{(2)}(t)-\beta_{o} F^{(1)}(U(1, t)) \\
& -\sum_{k=1}^{N} \beta_{k}\left[F^{(1)}(U(1, t))-\alpha_{k}^{2} F(U(1, t))+\alpha_{k}^{4} I_{k}(t)\right] \\
\vdots & \vdots \\
\vdots & \\
-\sum_{k=1}^{N} \beta_{k}\left[\sum_{i=0}^{(m)}(1, t)=\Phi^{(m)}(t)-\beta_{0} F^{(m-1)}(U(1, t))\right. &
\end{aligned}
$$


In general, the Nth order approximation of surface temperature $\mathrm{U}(1, \mathrm{t})$ is determined by assuming that $\mathrm{k}(\mathrm{t}-\tau)$ in (3.1) takes the form of (3.4), in which only the first $\mathrm{N}$ terms of the infinite sum are considered, such that $U(1, t)$ is approximated by (3.6). Then, by performing $N+1$ differentiations of (3.6), the resulting system of integrodifferential equations obtained by substituting $1, \ldots, \mathrm{N}+1$ for $\mathrm{m}$ in (3.8) are found to be

$$
\begin{aligned}
& U^{(1)}(1, t)= \Phi^{(1)}(t)-\beta_{o} F(U(1, t)) \\
&-\sum_{k=1}^{N} \beta_{k}\left[F(U(1, t))-\alpha_{k}^{2} I_{k}(t)\right] \\
& U^{(2)}(1, t)= \Phi^{(2)}(t)-\beta_{o} F^{(1)}(U(1, t)), \\
&-\sum_{k=1}^{N} \beta_{k}\left[F^{(1)}(U(1, t))-\alpha_{k}^{2} F(U(1, t))+\alpha_{k}^{4} I_{k}(t)\right],
\end{aligned}
$$

$$
\begin{gathered}
\vdots \\
\vdots \\
\vdots \\
U^{(N)}(1, t)=\Phi^{(N)}(t)-\beta_{0} F^{(N-1)}(U(1, t)) \\
-\sum_{k=1}^{N} \beta_{k}\left[\sum_{i=0}^{N-1}(-1)^{i} \alpha_{k}^{2 i} F^{(N-1-i)}(U(1, t))+(-1)^{N} \alpha_{k}^{2 N} I_{k}(t)\right] \\
-\sum_{k=1}^{N} \beta_{k}\left[\sum_{i=0}^{N}(-1)^{i} \alpha_{k}^{2 i} F^{(N-i)}(U(1, t))+(-1)^{N+1} \alpha_{k}^{2 N+2} I_{k}(t)\right]
\end{gathered}
$$


To eliminate integrals, $I_{k}(t)$, where $k=1, \ldots, N$, from $(3.12)$, consider the first $\mathrm{N}$ derivatives of the surface temperature which are given by (3.9)-(3.11). Rearrangement of the terms in (3.9)-(3.11) yields

$$
\begin{aligned}
& \sum_{k=1}^{N} \alpha_{k}^{2} \beta_{k} I_{k}(t)=U^{(1)}(1, t)-\Phi^{(1)}(t)+\beta_{o} F(U(1, t)) \\
& +\sum_{k=1}^{N} \beta_{k} F(U(1, t)) \\
& -\sum_{k=1}^{N} \alpha_{k}^{4} \beta_{k} I_{k}(t)=U^{(2)}(1, t)-\Phi^{(2)}(t)+\beta_{0} F^{(1)}(U(1, t)) \\
& +\sum_{k=1}^{N} \beta_{k}\left[F^{(1)}(U(1, t), t)-\alpha_{k}^{2} F(U(1, t))\right] \\
& (-1)^{N} \sum_{k=1}^{N} \beta_{k} \alpha_{k}^{2 N-2} I_{k}(i)=U^{(N-1)}(1, t)-\Phi^{(N-1)}(t)+\beta_{o} F^{(N-2)}(U(1, t)) \\
& +\sum_{k=1}^{N} \beta_{k}\left[\sum_{i=0}^{N-2}(-1)^{i} \alpha_{k}^{2 i} F^{(N-2-i)}(U(1, t))\right] \\
& (-1)^{N+1} \sum_{k=1}^{N} \beta_{k} \alpha_{k}^{2 N} I_{k}(t)=U^{(N)}(1, t)-\Phi^{(N)}(t)+\beta_{0} F^{(N-1)}(U(1, t)) \\
& +\sum_{k=1}^{N} \beta_{k}\left[\sum_{i=0}^{N-1}(-1)^{i} \alpha_{k}^{2 i} F^{(N-1-i)}(U(1, t))\right]
\end{aligned}
$$


In matrix representation,

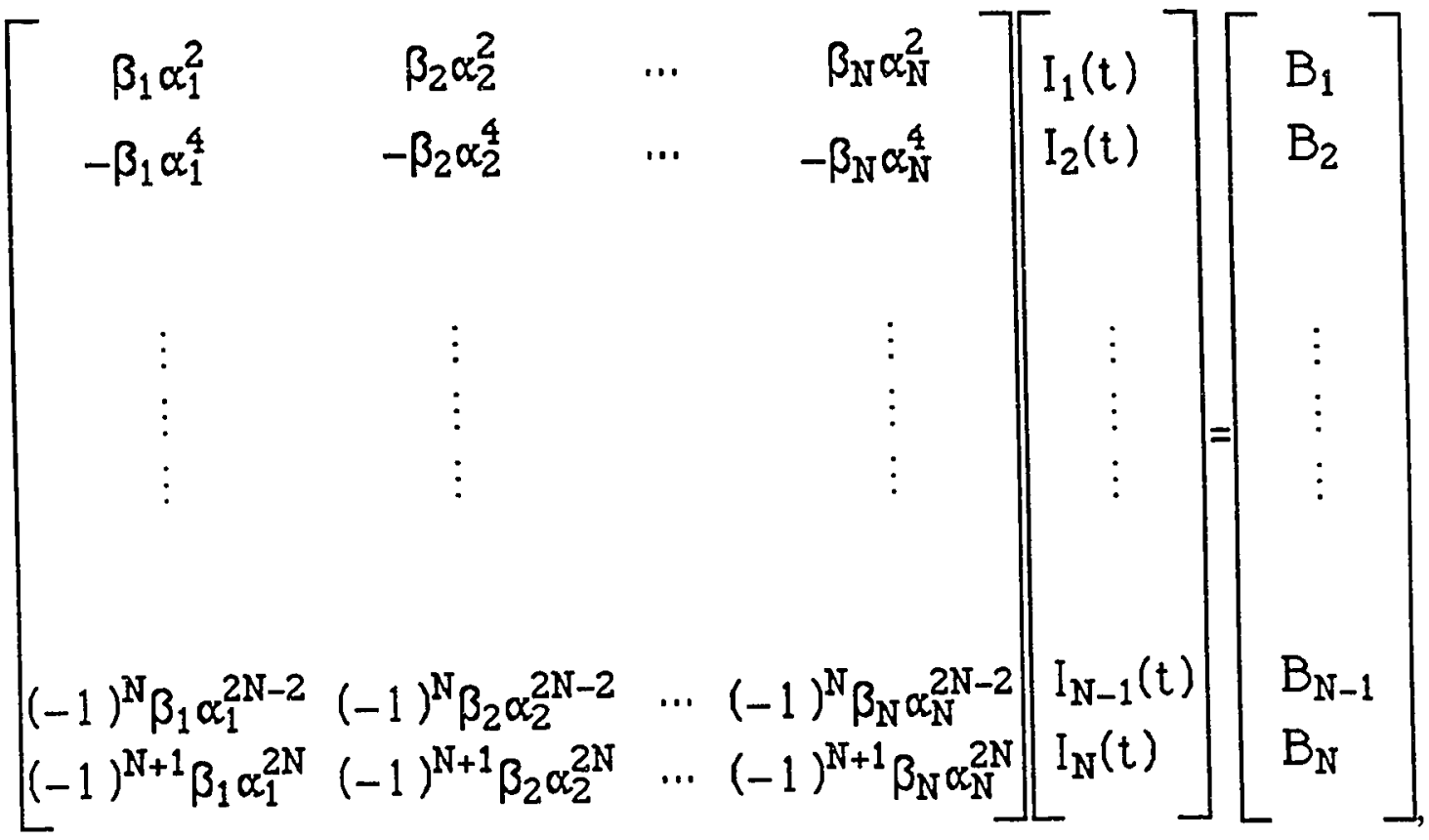

where $B_{1}, \ldots, B_{N}$ are defines as:

$$
\begin{aligned}
B_{1}=U^{(1)}(1, t)-\Phi^{(1)}(t)+\beta_{0} F(U(1, t)) & \\
& +\sum_{k=1}^{N} \beta_{k} F(U(1, t)) \\
B_{2}= & U^{(2)}(1, t)-\Phi^{(2)}(t)+\beta_{0} F^{(1)}(U(1, t)) \\
& +\sum_{k=1}^{N} \beta_{k}\left[F^{(1)}(U(1, t), t)-\alpha_{k}^{2} F(U(1, t))\right]
\end{aligned}
$$




$$
\begin{aligned}
B_{N-1} & =U^{(N-1)}(1, t)-\Phi^{(N-1)}(t)+\beta_{o} F^{(N-2)}(U(1, t)) \\
& +\sum_{k=1}^{N} \beta_{k}\left[\sum_{i=0}^{N-2}(-1)^{i} \alpha_{k}^{2 i} F^{(N-2-i)}(U(1, t))\right] \\
B_{N}= & U^{(N)}(1, t)-\Phi^{(N)}(t)+\beta_{o} F^{(N-1)}(U(1, t)) \\
& +\sum_{k=1}^{N} \beta_{k}\left[\sum_{i=0}^{N-1}(-1)^{i} \alpha_{k}^{2 i} F^{(N-1-i)}(U(1, t))\right]
\end{aligned}
$$

Now, let

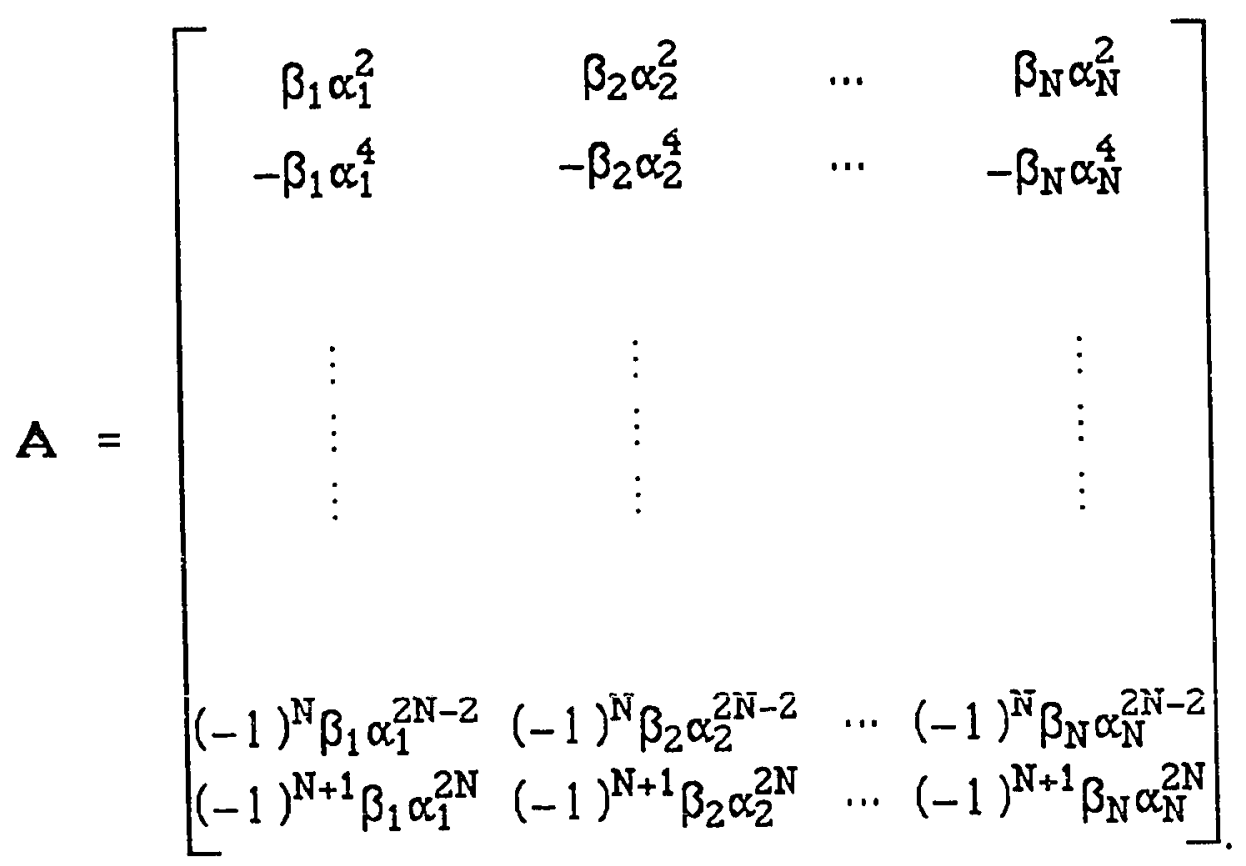

By Cramer's rule, $I_{1}(t), \ldots, I_{N}(t)$ can be expressed as a quotient of $N \times N$ determinants and are given by 


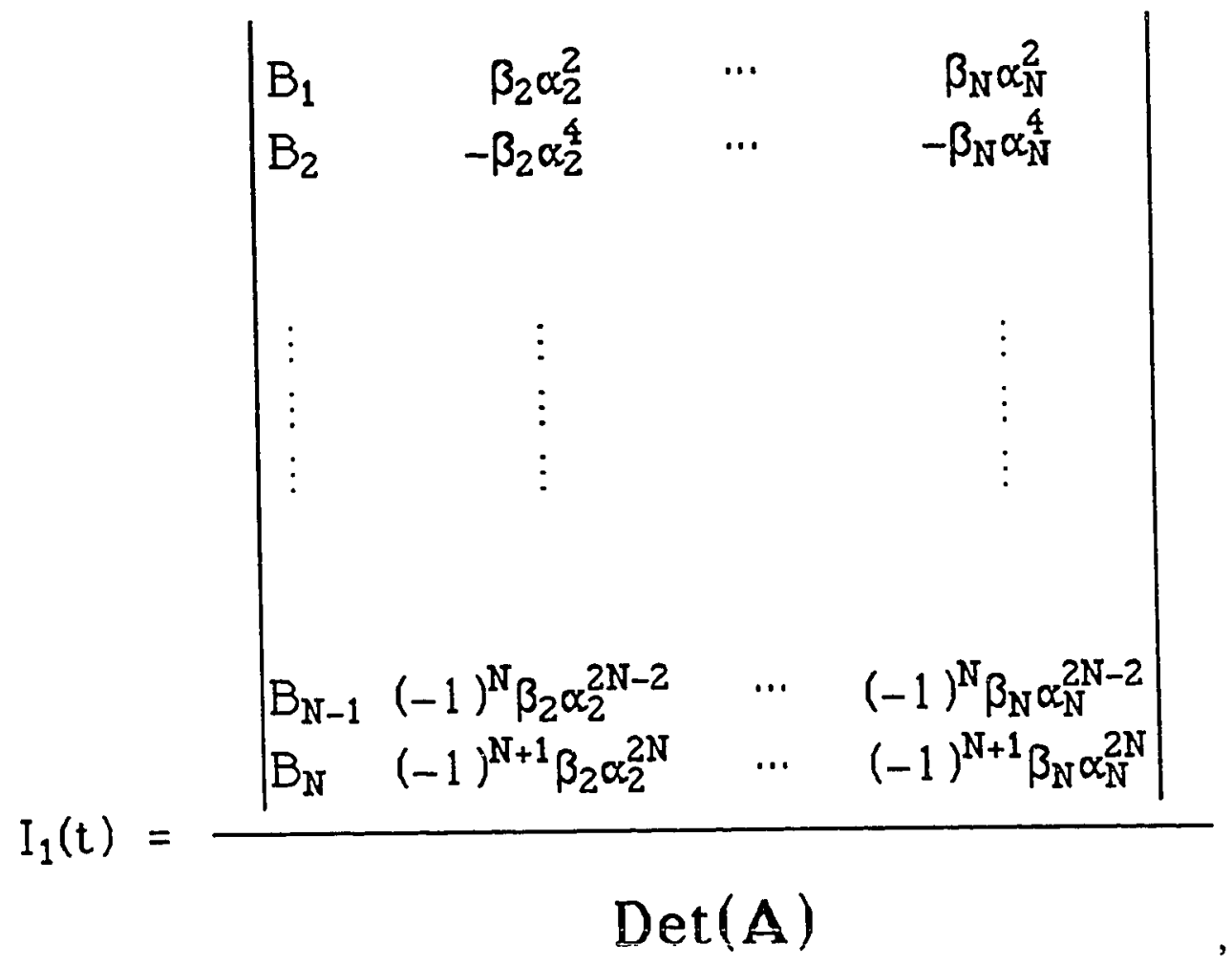

(3.23) 


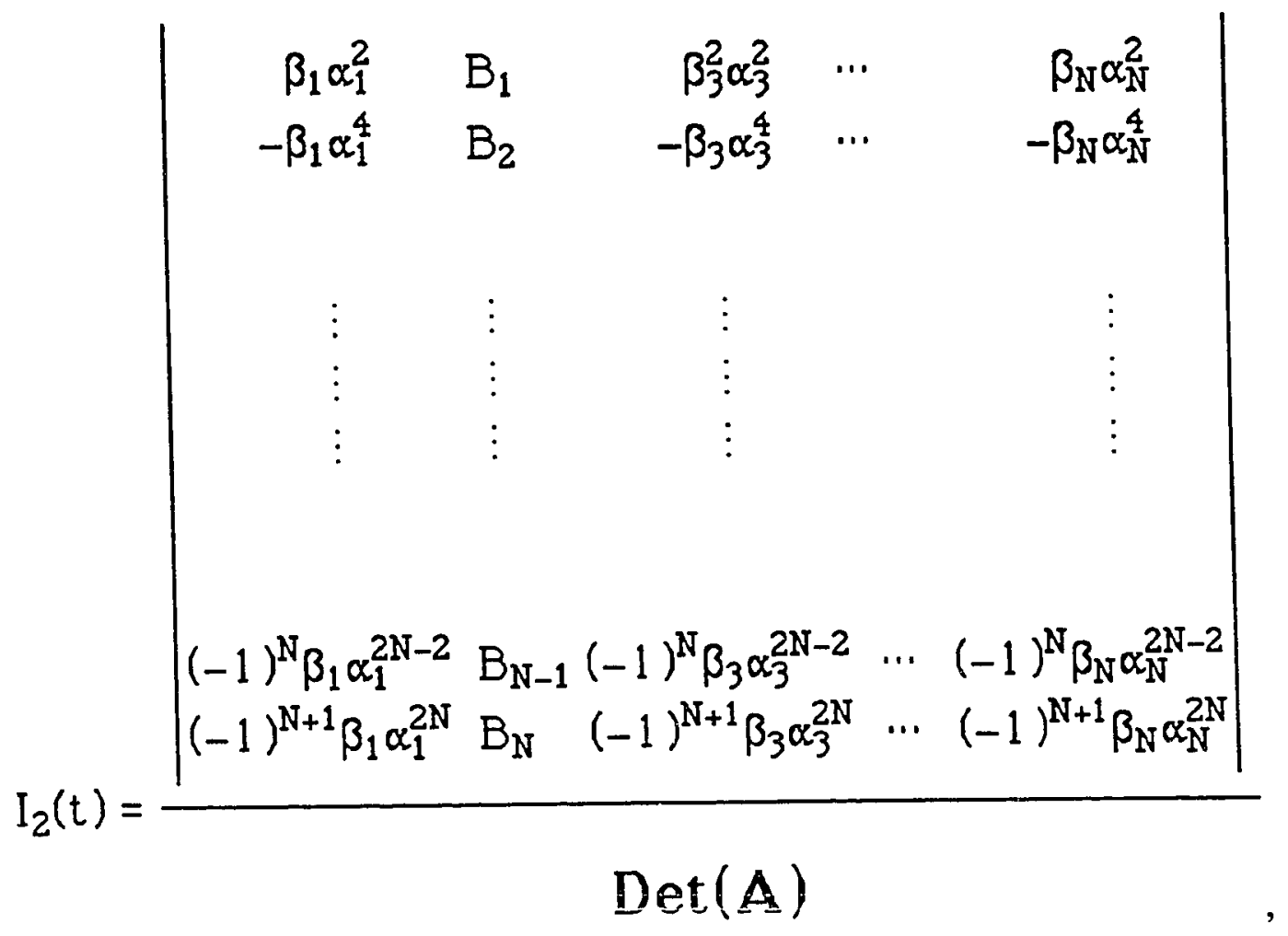

(3.24) 


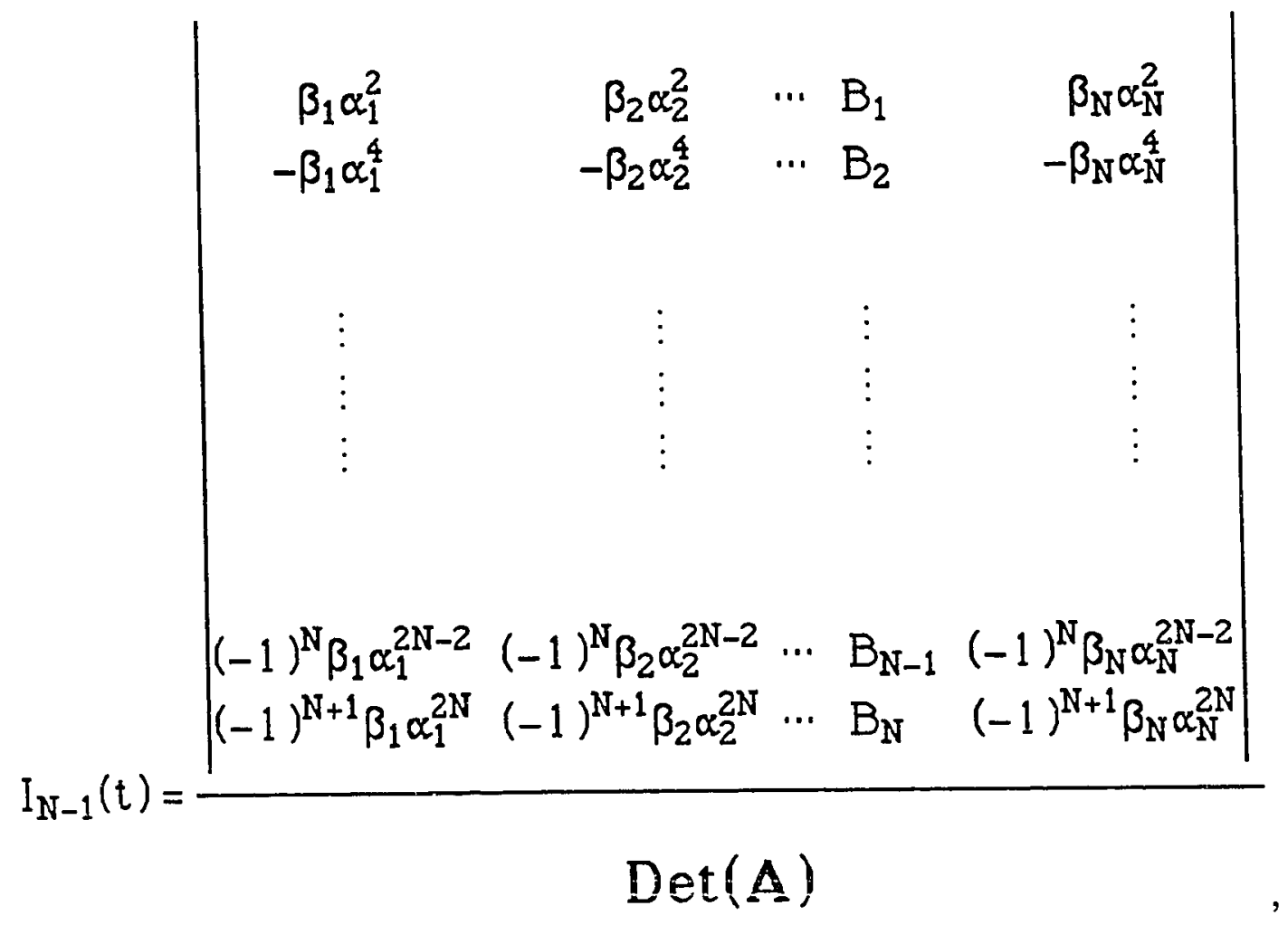

(3.25) 


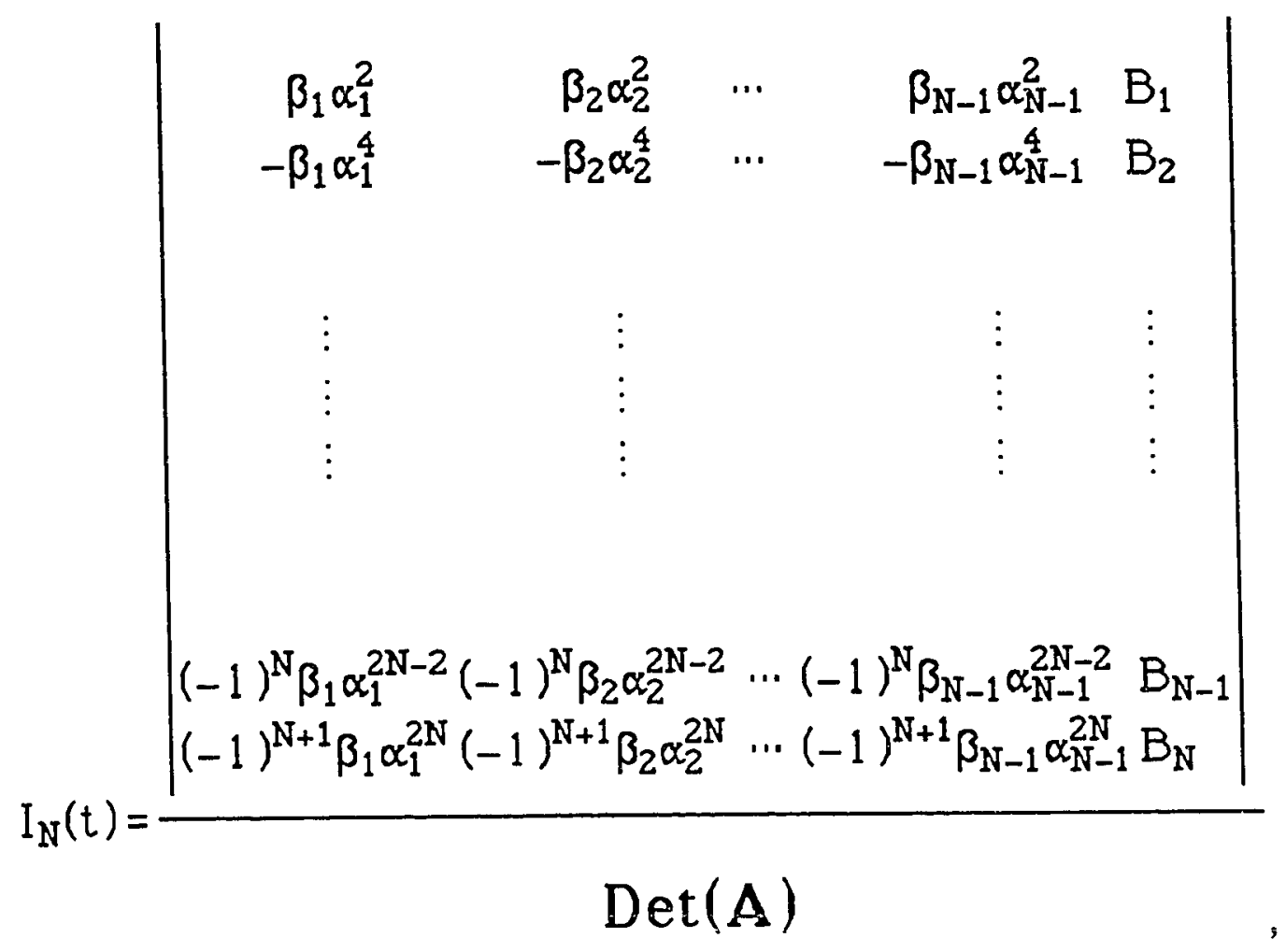

(3.26)

where 


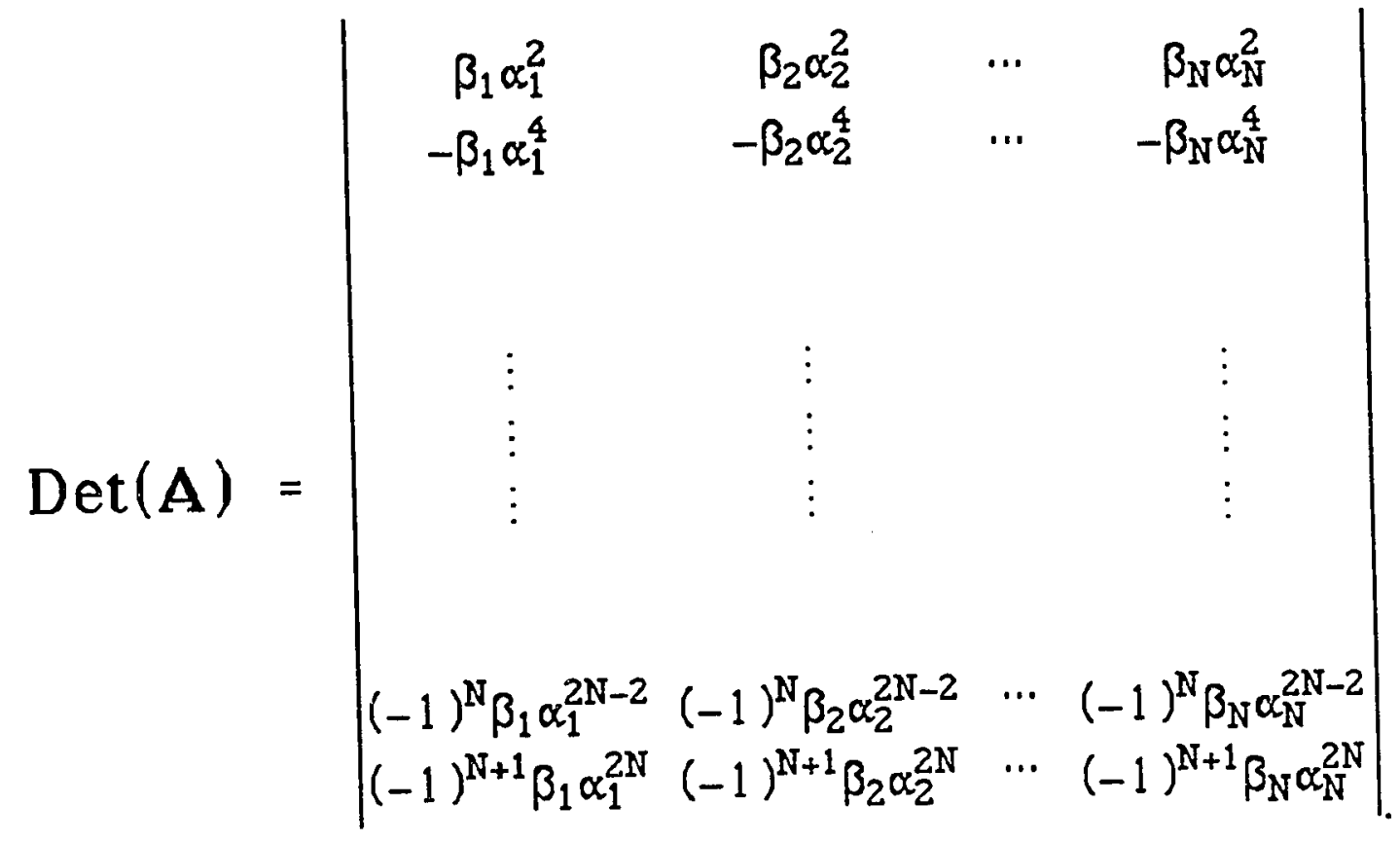

Next, by using the fundamental properties of determinants, (3.23), ..., (3.26) can be simplified as a quotient of $(\mathrm{N}-1) \times(\mathrm{N}-1)$ determinants: 
Define $\operatorname{Det}\left(\mathrm{A}^{\prime}\right)$ to be

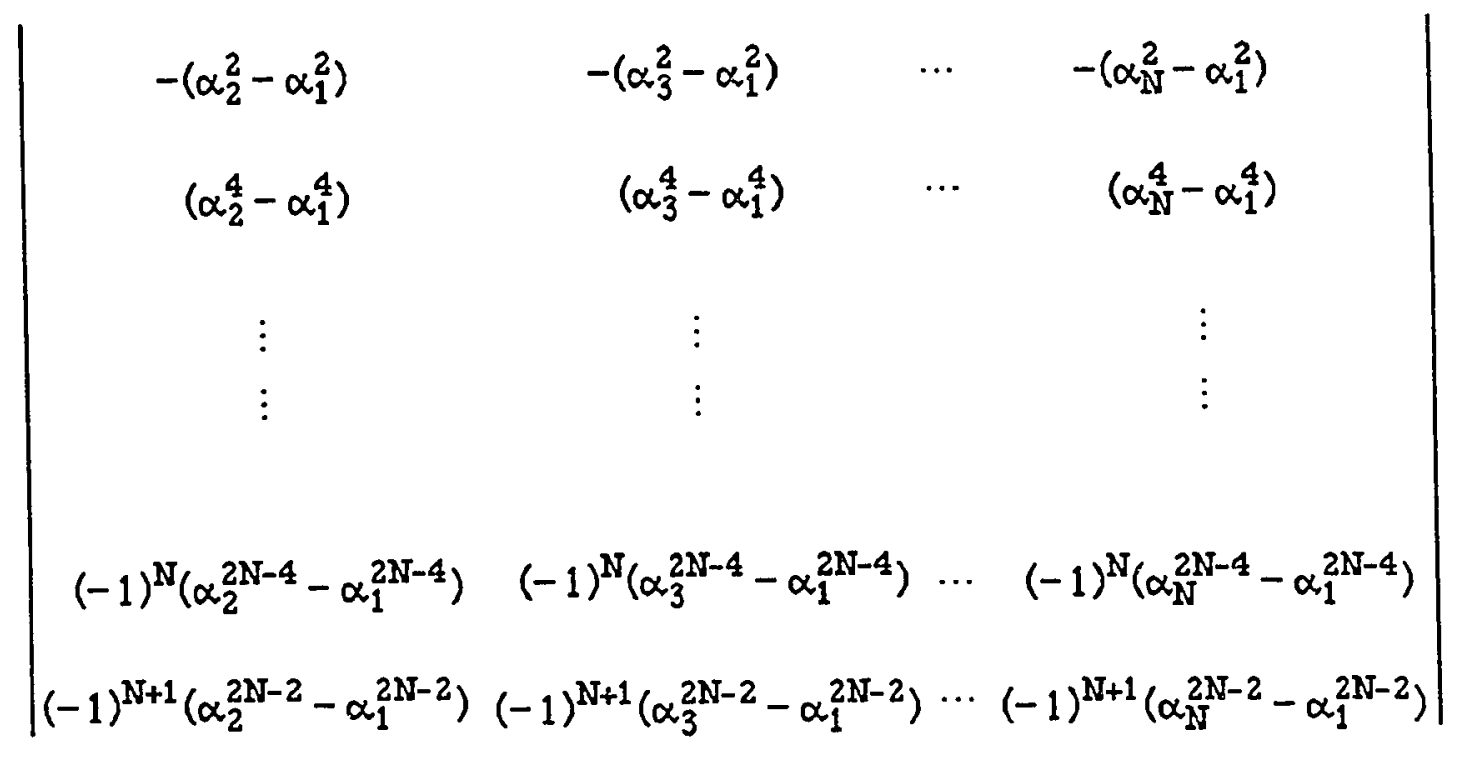

The formulas are found to be: 


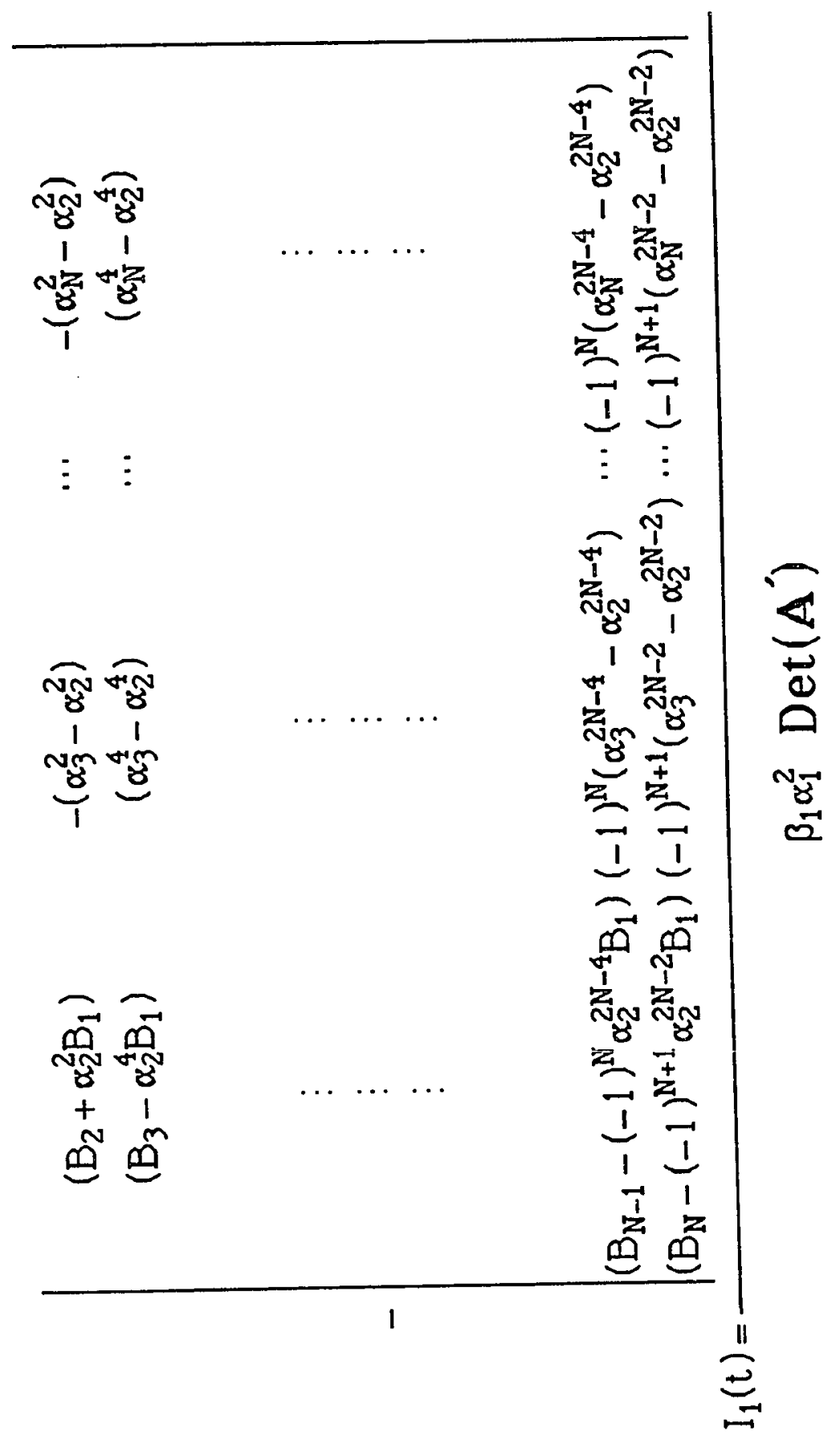


and for $k=2, \ldots, N-1$,

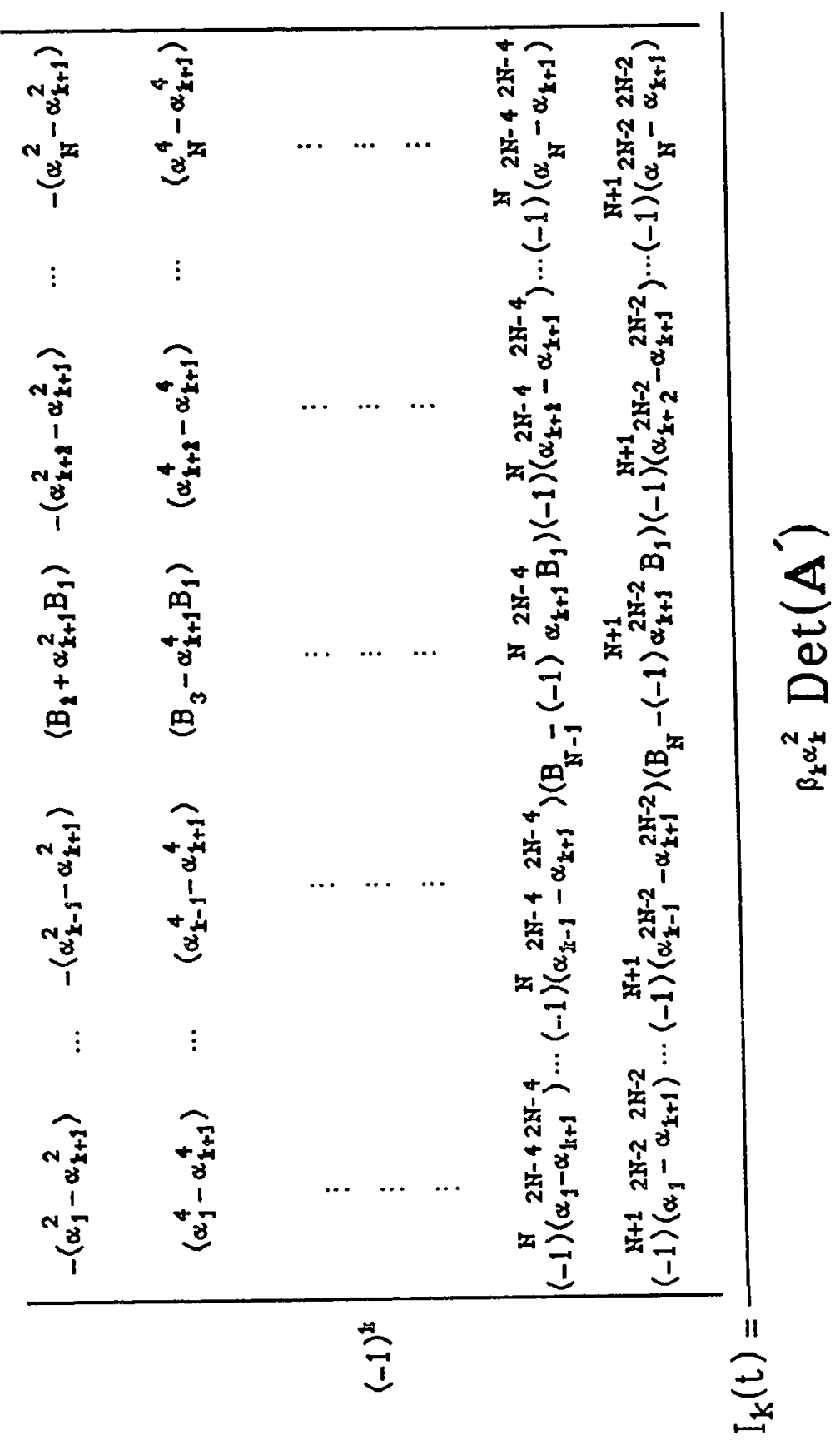


and

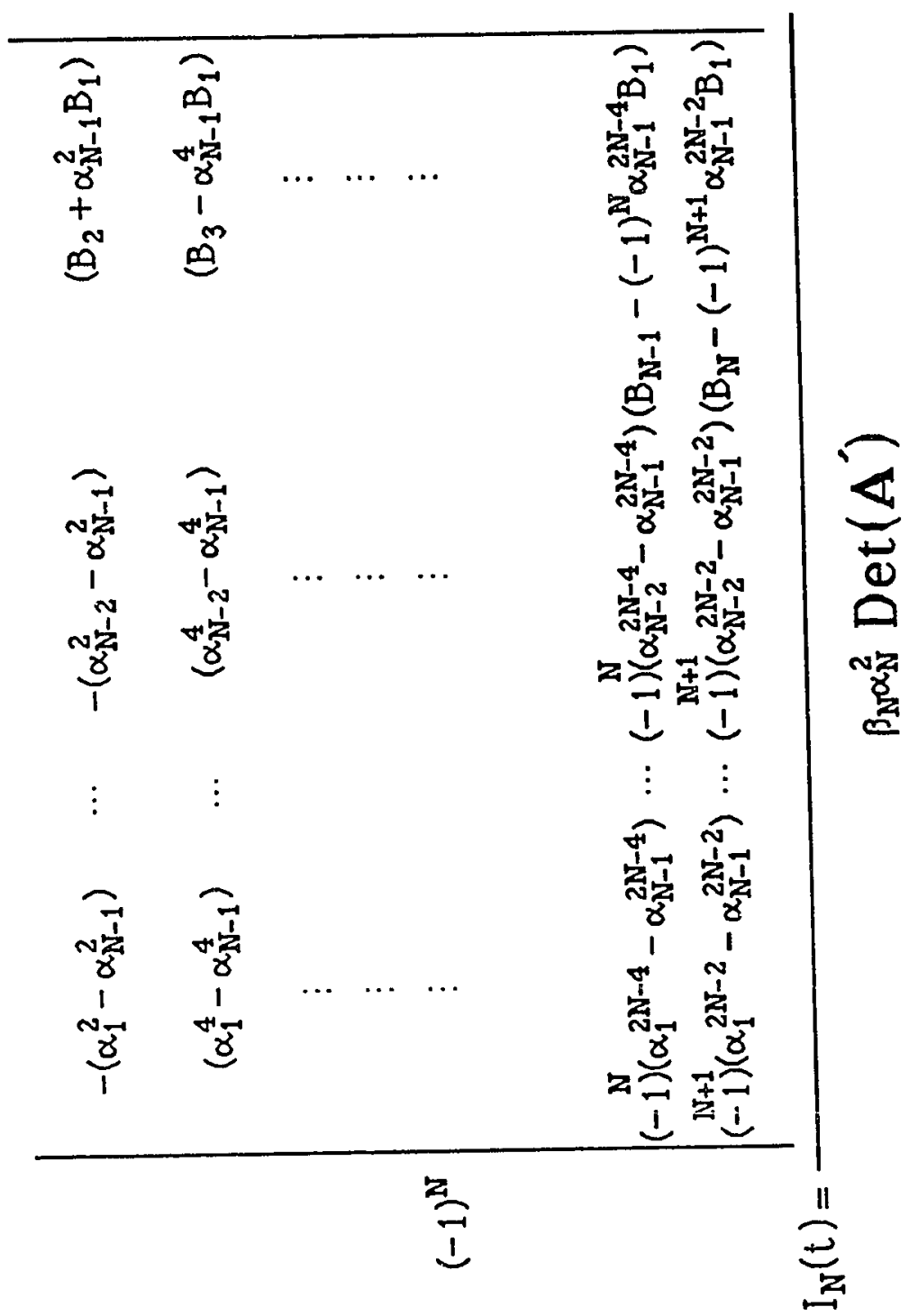

(3.31)

Thus, $I_{k}(t)$ where $k=1, \ldots, N$ in equation (3.12) can be determined explicitly by formulas (3.29)-(3.31). Hence, the integrodifferential equation (3.12) is 
reduced to a $\mathrm{N}+1$ th order nonlinear ordinary differential equation and is given by

$$
\begin{aligned}
& U^{(N+1)}(1, t)=\Phi^{(N+1)}(t)-\beta_{0} F^{(N)}(U(1, t)) \\
& -\sum_{k=1}^{N}(-1)^{i}\left(\sum_{i=0}^{N} \beta_{k} \alpha_{k}^{2 i}\right) F^{(N-i)}(U(1, t))-\sum_{k=1}^{N}(-1)^{N+1} \beta_{k} \alpha_{k}^{2 N+2} I_{k}(t)
\end{aligned}
$$

with the initial values

$$
\begin{aligned}
& U(1,0)=\Phi(0) \\
& U^{(1)}(1,0)=\Phi^{(1)}(0)-\beta_{0} F(U(1,0))-\sum_{k=1}^{N} \beta_{k} F(U(1,0)) \\
& U^{(2)}(1,0)= \Phi^{(2)}(0)-\beta_{0} F^{(1)}(U(1,0)) \\
&-\sum_{k=1}^{N} \beta_{k}\left[F^{(1)}(U(1,0))-\alpha_{k}^{2} F(U(1,0))\right] \\
& \vdots \\
& \vdots \\
& \vdots \\
& U^{(N)}(1,0)= \Phi^{(N)}(0)-\beta_{0} F^{(N-1)}(U(1,0)) \\
&-\sum_{i=0}^{N-1}(-1)^{i} F^{(N-1-i)}(U(1,0))\left(\sum_{k=1}^{N} \beta_{k} \alpha_{k}^{2 i}\right)
\end{aligned}
$$

which can be obtained by putting $t=0$ in $(3.9)-(3.12)$, and $I_{1}(t), \ldots, I_{N}(t)$ are determined by formulas (3.29)-(3.31). 
The Runge-Kutta method is then applied to the nonlinear ordinary differential equation (3.32) with initial conditions (3.33)-(3.36) for the solution. Hence, the Nth order approximation of the surface temperature will be given by the numerical solution of a $\mathbf{N}+1$ th order nonlinear ordinary differential equation.

As an example, in the next section, formulas for the third and the fifth order approximation of the method will be presented. Details of the derivation of the equations will not be produced, and only major results will be given.

\subsection{The Third Order Approximation}

The third order approximation of the surface temperature of (3.1) is given by the numerical solution of the following fourth order nonlinear ordinary differential equation:

$$
\begin{aligned}
U^{(4)}(1, t)= & \Phi^{(4)}(t)-\left(\beta_{0}+\beta_{1}+\beta_{2}+\beta_{3}\right) F^{(3)}(U(1, t)) \\
& +\left(\beta_{1} \alpha_{1}^{2}+\beta_{2} \alpha_{2}^{2}+\beta_{3} \alpha_{3}^{2}\right) F^{(2)}(U(1, t)) \\
& -\left(\beta_{1} \alpha_{1}^{4}+\beta_{2} \alpha_{2}^{4}+\beta_{3} \alpha_{3}^{4}\right) F^{(1)}(U(1, t)) \\
& +\left(\beta_{1} \alpha_{1}^{6}+\beta_{2} \alpha_{2}^{6}+\beta_{3} \alpha_{3}^{6}\right) F(U(1, t)) \\
& -\left(\beta_{1} \alpha_{1}^{8} I_{1}(t)+\beta_{2} \alpha_{2}^{8} I_{2}(t)+\beta_{3} \alpha_{3}^{8} I_{3}(t)\right)
\end{aligned}
$$

with initial conditions

$$
\begin{aligned}
& U(1,0)=\Phi(0) \\
& \begin{aligned}
U^{(1)}(1,0)= & \Phi^{(1)}(0)-\left(\beta_{0}+\beta_{1}+\beta_{2}+\beta_{3}\right) F(\Phi(0)) \\
U^{(2)}(1,0)= & \Phi^{(2)}(0)-\left(\beta_{0}+\beta_{1}+\beta_{2}+\beta_{3}\right) F^{(1)}(\Phi(0)) \\
& +\left(\beta_{1} \alpha_{1}^{2}+\beta_{2} \alpha_{2}^{2}+\beta_{3} \alpha_{3}^{2}\right) F(\Phi(0))
\end{aligned}
\end{aligned}
$$




$$
\begin{aligned}
U^{(3)}(1,0)= & \Phi^{(3)}(0)-\left(\beta_{0}+\beta_{1}+\beta_{2}+\beta_{3}\right) F^{(2)}(\Phi(0)) \\
& +\left(\beta_{1} \alpha_{1}^{2}+\beta_{2} \alpha_{2}^{2}+\beta_{3} \alpha_{3}^{2}\right) F^{(1)}(\Phi(0)) \\
& -\left(\beta_{1} \alpha_{1}^{4}+\beta_{2} \alpha_{2}^{4}+\beta_{3} \alpha_{3}^{4}\right) F(\Phi(0))
\end{aligned}
$$

$I_{1}(t), I_{2}(t)$, and $I_{3}(t)$ in (3.37) are found to be

$$
\begin{aligned}
& I_{1}(t)=\frac{-\left|\begin{array}{rr}
\left(B_{2}+\alpha_{2}^{2} B_{1}\right) & -\left(\alpha_{3}^{2}-\alpha_{2}^{2}\right) \\
\left(B_{3}-\alpha_{2}^{4} B_{1}\right) & \left(\alpha_{3}^{4}-\alpha_{2}^{4}\right)
\end{array}\right|}{\beta_{1} \alpha_{1}^{2} \operatorname{Det}\left(A^{\prime}\right)}, \\
& I_{2}(t)=\frac{\left|\begin{array}{ll}
\left(\alpha_{1}^{2}-\alpha_{3}^{2}\right) & \left(B_{2}+\alpha_{3}^{2} B_{1}\right) \\
\left(\alpha_{1}^{4}-\alpha_{3}^{4}\right) & \left(B_{3}-\alpha_{3}^{4} B_{1}\right)
\end{array}\right|}{\beta_{2} \alpha_{2}^{2} \operatorname{Det}\left(A^{\prime}\right)} \\
& I_{3}(t)=\frac{-\left|\begin{array}{cc}
\left(\alpha_{1}^{2}-\alpha_{2}^{2}\right) & \left(B_{2}+\alpha_{2}^{2} B_{1}\right) \\
\left(\alpha_{1}^{4}-\alpha_{2}^{4}\right) & \left(B_{3}-\alpha_{2}^{4} B_{1}\right)
\end{array}\right|}{\beta_{3} \alpha_{3}^{2} \overline{D e t}\left(A^{\prime}\right)},
\end{aligned}
$$

where

$$
\begin{aligned}
B_{1}= & U^{(1)}(1, t)-\Phi^{(1)}(t)+\left(\beta_{0}+\beta_{1}+\beta_{2}+\beta_{3}\right) F(U(1, t)), \\
B_{2}= & U^{(2)}(1, t)-\Phi^{(2)}(t)+\left(\beta_{0}+\beta_{1}+\beta_{2}+\beta_{3}\right) F^{(1)}(U(1, t)) \\
& -\left(\beta_{1} \alpha_{1}^{2}+\beta_{2} \alpha_{2}^{2}+\beta_{3} \alpha_{3}^{2}\right) F(U(1, t)),
\end{aligned}
$$




$$
\begin{gathered}
B_{3}=U^{(3)}(1, t)-\Phi^{(3)}(t)+\left(\beta_{0}+\beta_{1}+\beta_{2}+\beta_{3}\right) F^{(2)}(U(1, t)) \\
-\left(\beta_{1} \alpha_{1}^{2}+\beta_{2} \alpha_{2}^{2}+\beta_{3} \alpha_{3}^{2}\right) F^{(1)}(U(1, t))+\left(\beta_{1} \alpha_{1}^{4}+\beta_{2} \alpha_{2}^{4}+\beta_{3} \alpha_{3}^{4}\right) F(U(1, t)),
\end{gathered}
$$

and

$$
\operatorname{Det}\left(\mathbf{A}^{\prime}\right)=\left|\begin{array}{rr}
-\left(\alpha_{2}^{2}-\alpha_{1}^{2}\right) & -\left(\alpha_{3}^{2}-\alpha_{1}^{2}\right) \\
\left(\alpha_{2}^{4}-\alpha_{1}^{4}\right) & \left(\alpha_{3}^{4}-\alpha_{1}^{4}\right)
\end{array}\right| .
$$

\subsection{The Fifth Order Approximation}

The fifth order approximation of the surface temperature of (3.1) is given by the numerical solution of the following sixth order nonlinear ordinary differential equation:

$$
\begin{aligned}
U^{(6)}(1, t)=\Phi^{(6)}(t)-\left(\beta_{0}+\beta_{1}+\beta_{2}+\beta_{3}+\beta_{4}+\beta_{5}\right) F^{(5)}(U(1, t)) \\
+\left(\beta_{1} \alpha_{1}^{2}+\beta_{2} \alpha_{2}^{2}+\beta_{3} \alpha_{3}^{2}+\beta_{4} \alpha_{4}^{2}+\beta_{5} \alpha_{5}^{2}\right) F^{(4)}(U(1, t)) \\
-\left(\beta_{1} \alpha_{1}^{4}+\beta_{2} \alpha_{2}^{4}+\beta_{3} \alpha_{3}^{4}+\beta_{4} \alpha_{4}^{4}+\beta_{5} \alpha_{5}^{4}\right) F^{(3)}(U(1, t)) \\
+\left(\beta_{1} \alpha_{1}^{6}+\beta_{2} \alpha_{2}^{6}+\beta_{3} \alpha_{3}^{6}+\beta_{4} \alpha_{4}^{6}+\beta_{5} \alpha_{5}^{6}\right) F^{(2)}(U(1, t)) \\
-\left(\beta_{1} \alpha_{1}^{8}+\beta_{2} \alpha_{2}^{8}+\beta_{3} \alpha_{3}^{8}+\beta_{4} \alpha_{4}^{8}+\beta_{5} \alpha_{5}^{8}\right) F^{(1)}(U(1, t)) \\
+\left(\beta_{1} \alpha_{1}^{10}+\beta_{2} \alpha_{2}^{10}+\beta_{3} \alpha_{3}^{10}+\beta_{4} \alpha_{4}^{10}+\beta_{5} \alpha_{5}^{10}\right) F(U(1, t)) \\
-\left(\beta_{1} \alpha_{1}^{12} I_{1}(t)+\beta_{2} \alpha_{2}^{12} I_{2}(t)+\beta_{3} \alpha_{3}^{12} I_{3}(t)+\beta_{4} \alpha_{1}^{12} I_{4}(t)+\beta_{5} \alpha_{2}^{12} I_{5}(t)\right)
\end{aligned}
$$

with initial conditions

$$
\begin{aligned}
& U(1,0)=\Phi(0), \\
& U^{(1)}(1,0)=\Phi^{(1)}(0)-\left(\beta_{0}+\beta_{1}+\beta_{2}+\beta_{3}+\beta_{4}+\beta_{5}\right) F(\Phi(0)), \\
& U^{(2)}(1,0)=\Phi^{(2)}(0)-\left(\beta_{0}+\beta_{1}+\beta_{2}+\beta_{3}+\beta_{4}+\beta_{5}\right) F^{(1)}(\Phi(0))
\end{aligned}
$$




$$
\begin{aligned}
&+\left(\beta_{1} \alpha_{1}^{2}+\beta_{2} \alpha_{2}^{2}+\beta_{3} \alpha_{3}^{2}+\beta_{4} \alpha_{4}^{2}+\beta_{5} \alpha_{5}^{2}\right) F(\Phi(0)) \\
& U^{(3)}(1,0)=\Phi^{(3)}(0)-\left(\beta_{0}+\beta_{1}+\beta_{2}+\beta_{3}+\beta_{4}+\beta_{5}\right) F^{(2)}(\Phi(0)) \\
&+\left(\beta_{1} \alpha_{1}^{2}+\beta_{2} \alpha_{2}^{2}+\beta_{3} \alpha_{3}^{2}+\beta_{4} \alpha_{4}^{2}+\beta_{5} \alpha_{5}^{2}\right) F^{(1)}(\Phi(0)) \\
&-\left(\beta_{1} \alpha_{1}^{4}+\beta_{2} \alpha_{2}^{4}+\beta_{3} \alpha_{3}^{4}+\beta_{4} \alpha_{4}^{4}+\beta_{5} \alpha_{5}^{4}\right) F(\Phi(0)) \\
& U^{(4)}(1,0)=\Phi^{(4)}(0)-\left(\beta_{0}+\beta_{1}+\beta_{2}+\beta_{3}+\beta_{4}+\beta_{5}\right) F^{(3)}(\Phi(0)) \\
&+\left(\beta_{1} \alpha_{1}^{2}+\beta_{2} \alpha_{2}^{2}+\beta_{3} \alpha_{3}^{2}+\beta_{4} \alpha_{4}^{2}+\beta_{5} \alpha_{5}^{2}\right) F^{(2)}(\Phi(0)) \\
&-\left(\beta_{1} \alpha_{1}^{4}+\beta_{2} \alpha_{2}^{4}+\beta_{3} \alpha_{3}^{4}+\beta_{4} \alpha_{4}^{4}+\beta_{5} \alpha_{5}^{4}\right) F^{(1)}(\Phi(0)) \\
&+\left(\beta_{1} \alpha_{1}^{6}+\beta_{2} \alpha_{2}^{6}+\beta_{3} \alpha_{3}^{6}+\beta_{4} \alpha_{4}^{6}+\beta_{5} \alpha_{5}^{6}\right) F(\Phi(0)) \\
& U^{(5)}(1,0)=\Phi \\
&+\left(\beta_{1} \alpha_{1}^{2}+\beta_{2} \alpha_{2}^{2}+\beta_{3} \alpha_{3}^{2}+\beta_{4} \alpha_{4}^{2}+\beta_{5} \alpha_{5}^{2}\right) F^{(3)}(\Phi(0)) \\
&-\left(\beta_{1} \alpha_{1}^{4}+\beta_{2} \alpha_{2}^{4}+\beta_{3} \alpha_{3}^{4}+\beta_{4} \alpha_{4}^{4}+\beta_{5} \alpha_{5}^{4}\right) F^{(2)}(\Phi(0)) \\
&+\left(\beta_{1} \alpha_{1}^{6}+\beta_{2} \alpha_{2}^{6}+\beta_{3} \alpha_{3}^{6}+\beta_{4} \alpha_{4}^{6}+\beta_{5} \alpha_{5}^{6}\right) F^{(1)}(\Phi(0)) \\
&-\left(\beta_{1} \alpha_{1}^{8}+\beta_{2} \alpha_{2}^{8}+\beta_{3} \alpha_{3}^{8}+\beta_{4} \alpha_{4}^{8}+\beta_{5} \alpha_{5}^{8}\right) F(\Phi(0)) .
\end{aligned}
$$

$I_{1}(t), I_{2}(t), I_{3}(t), I_{4}(t)$, and $I_{5}(t)$ in (3.44) are found to be

$$
=\frac{-\left|\begin{array}{cccc}
\left(B_{2}+\alpha_{2}^{2} B_{1}\right) & -\left(\alpha_{3}^{2}-\alpha_{2}^{2}\right) & -\left(\alpha_{4}^{2}-\alpha_{2}^{2}\right) & -\left(\alpha_{5}^{2}-\alpha_{2}^{2}\right) \\
\left(B_{3}-\alpha_{2}^{4} B_{1}\right) & \left(\alpha_{3}^{4}-\alpha_{2}^{4}\right) & \left(\alpha_{4}^{4}-\alpha_{2}^{4}\right) & \left(\alpha_{5}^{4}-\alpha_{2}^{4}\right) \\
\left(B_{4}+\alpha_{2}^{6} B_{1}\right) & -\left(\alpha_{3}^{6}-\alpha_{2}^{6}\right) & -\left(\alpha_{4}^{6}-\alpha_{2}^{6}\right) & -\left(\alpha_{5}^{6}-\alpha_{2}^{6}\right) \\
\left(B_{5}-\alpha_{2}^{8} B_{1}\right) & \left(\alpha_{3}^{8}-\alpha_{2}^{8}\right) & \left(\alpha_{4}^{8}-\alpha_{2}^{8}\right) & \left(\alpha_{5}^{8}-\alpha_{2}^{8}\right)
\end{array}\right|}{\beta_{1} \alpha_{1}^{2} \operatorname{Det}\left(A^{\prime}\right)}
$$




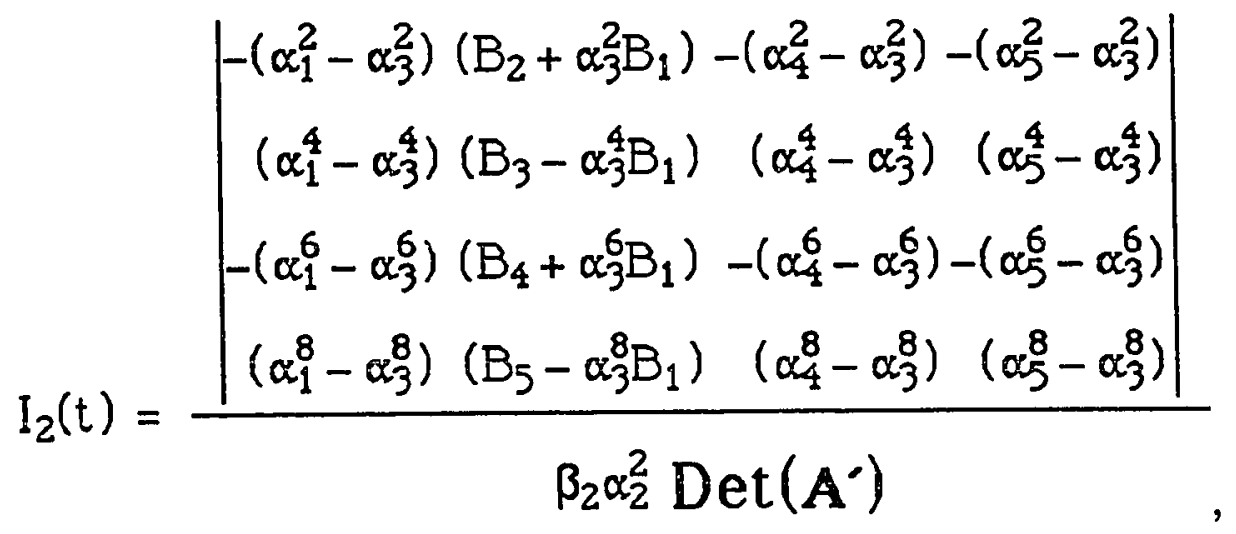

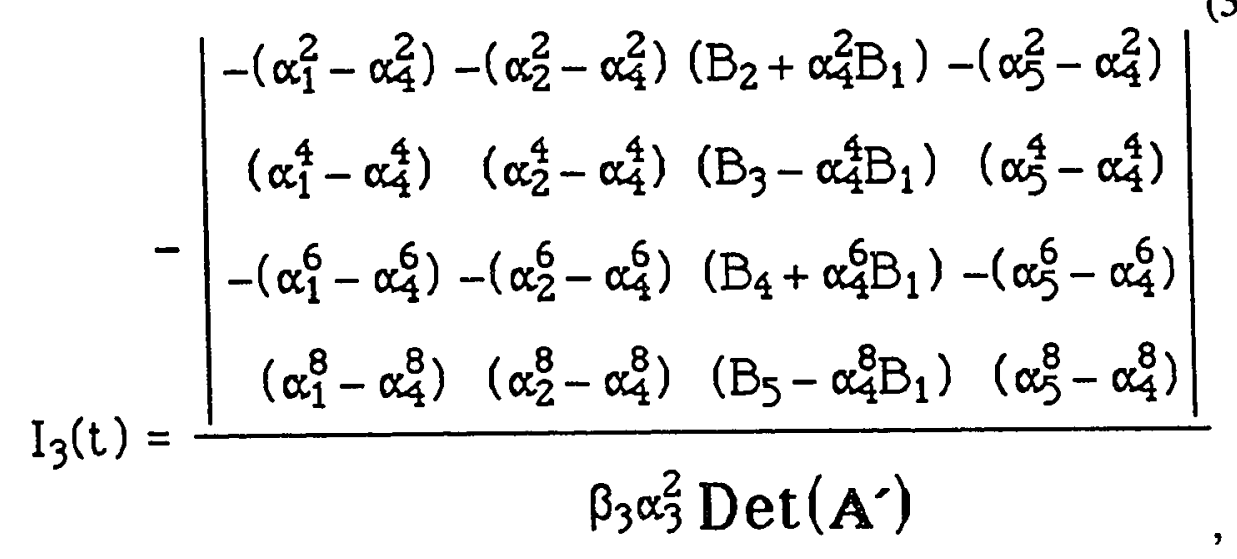

$I_{4}(t)=\frac{\left|\begin{array}{rrrr}-\left(\alpha_{1}^{2}-\alpha_{5}^{2}\right) & -\left(\alpha_{2}^{2}-\alpha_{5}^{2}\right) & -\left(\alpha_{3}^{2}-\alpha_{5}^{2}\right) & \left(B_{2}+\alpha_{5}^{2} B_{1}\right) \\ \left(\alpha_{1}^{4}-\alpha_{5}^{4}\right) & \left(\alpha_{2}^{4}-\alpha_{5}^{4}\right) & \left(\alpha_{3}^{4}-\alpha_{5}^{4}\right) & \left(B_{3}-\alpha_{5}^{4} B_{1}\right) \\ -\left(\alpha_{1}^{6}-\alpha_{5}^{6}\right) & \left(\alpha_{2}^{6}-\alpha_{5}^{6}\right) & -\left(\alpha_{3}^{6}-\alpha_{5}^{6}\right) & \left(B_{4}+\alpha_{5}^{6} B_{1}\right) \\ \left(\alpha_{1}^{8}-\alpha_{5}^{8}\right) & \left(\alpha_{2}^{8}-\alpha_{5}^{8}\right) & \left(\alpha_{3}^{8}-\alpha_{5}^{8}\right) & \left(B_{5}-\alpha_{5}^{8} B_{1}\right)\end{array}\right|}{\beta_{4} \alpha_{4}^{2} \operatorname{Det}\left(A^{0}\right)}$ 


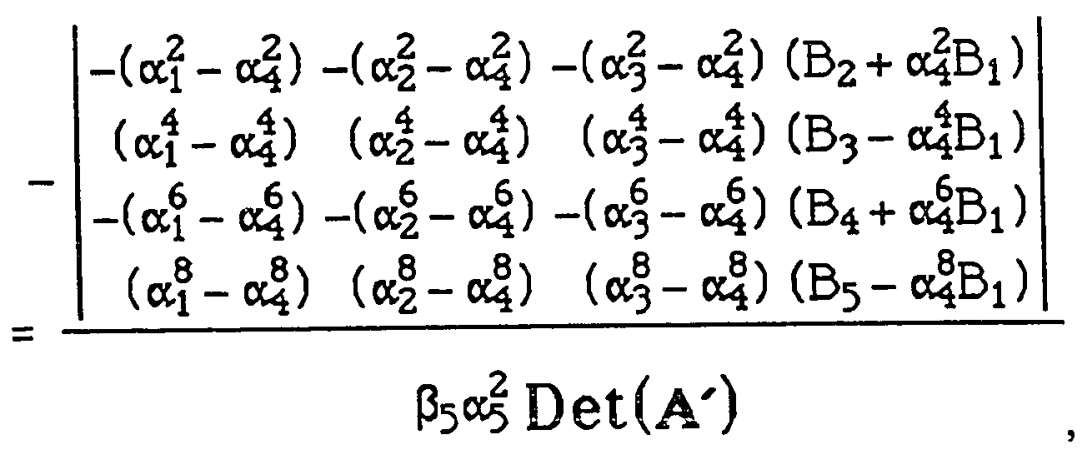

where

$$
\begin{aligned}
& \operatorname{Det}\left(\mathbf{A}^{-}\right)=\left|\begin{array}{rrrr}
-\left(\alpha_{2}^{2}-\alpha_{1}^{2}\right) & -\left(\alpha_{3}^{2}-\alpha_{1}^{2}\right) & -\left(\alpha_{4}^{2}-\alpha_{1}^{2}\right) & -\left(\alpha_{5}^{2}-\alpha_{1}^{2}\right) \\
\left(\alpha_{2}^{4}-\alpha_{1}^{4}\right) & \left(\alpha_{3}^{4}-\alpha_{1}^{4}\right) & \left(\alpha_{4}^{4}-\alpha_{1}^{4}\right) & \left(\alpha_{5}^{4}-\alpha_{1}^{4}\right) \\
-\left(\alpha_{2}^{6}-\alpha_{1}^{6}\right) & -\left(\alpha_{3}^{6}-\alpha_{1}^{6}\right) & -\left(\alpha_{4}^{6}-\alpha_{1}^{6}\right) & -\left(\alpha_{5}^{6}-\alpha_{1}^{6}\right) \\
\left(\alpha_{2}^{8}-\alpha_{1}^{8}\right) & \left(\alpha_{3}^{8}-\alpha_{1}^{8}\right) & \left(\alpha_{4}^{8}-\alpha_{1}^{8}\right) & \left(\alpha_{5}^{8}-\alpha_{1}^{8}\right)
\end{array}\right|, \\
& B_{1}=U^{(1)}(1, t)-\Phi^{(1)}(t)+\left(\beta_{0}+\beta_{1}+\beta_{2}+\beta_{3}\right) F(U(1, t)), \\
& B_{2}=U^{(2)}(1, t)-\Phi^{(2)}(t)+\left(\beta_{0}+\beta_{1}+\beta_{2}+\beta_{3}\right) F^{(1)}(U(1, t)) \\
& -\left(\beta_{1} \alpha_{1}^{2}+\beta_{2} \alpha_{2}^{2}+\beta_{3} \alpha_{3}^{2}\right) F(U(1, t)) \text {, } \\
& B_{3}=U^{(3)}(1, t)-\Phi^{(3)}(t)+\left(\beta_{0}+\beta_{1}+\beta_{2}+\beta_{3}+\beta_{\alpha}+\beta_{5}\right) F^{(2)}(U(1, t)) \\
& -\left(\beta_{1} \alpha_{1}^{2}+\beta_{2} \alpha_{2}^{2}+\beta_{3} \alpha_{3}^{2}+\beta_{4} \alpha_{4}^{2}+\beta_{5} \alpha_{5}^{2}\right) F^{(1)}(U(1, t)) \\
& +\left(\beta_{1} \alpha_{1}^{4}+\beta_{2} \alpha_{2}^{4}+\beta_{3} \alpha_{3}^{4}+\beta_{4} \alpha_{4}^{4}+\beta_{5} \alpha_{5}^{4}\right) F(U(1, t)) \text {. } \\
& B_{4}=U^{(\varepsilon)}(1, t)-\Phi^{(4)}(t)+\left(\beta_{0}+\beta_{1}+\beta_{2}+\beta_{3}+\beta_{4}+\beta_{5}\right) F^{(3)}(U(1, t)) \\
& -\left(\beta_{1} \alpha_{1}^{2}+\beta_{2} \alpha_{2}^{2}+\beta_{3} \alpha_{3}^{2}+\beta_{4} \alpha_{4}^{2}+\beta_{5} \alpha_{5}^{2}\right) F^{(2)}(U(1, t)) \\
& +\left(\beta_{1} \alpha_{1}^{4}+\beta_{2} \alpha_{2}^{4}+\beta_{3} \alpha_{3}^{4}+\beta_{4} \alpha_{4}^{4}+\beta_{5} \alpha_{5}^{4}\right) F^{(1)}(U(1, t)) \\
& -\left(\beta_{1} \alpha_{1}^{6}+\beta_{2} \alpha_{2}^{6}+\beta_{3} \alpha_{3}^{6}+\beta_{4} \alpha_{4}^{6}+\beta_{5} \alpha_{5}^{6}\right) F(U(1, \ell)) \text {. }
\end{aligned}
$$


and

$$
\begin{aligned}
& B_{5}=U^{(5)}(1, t)-\Phi^{(5)}(t)+\left(\beta_{0}+\beta_{1}+\beta_{2}+\beta_{3}+\beta_{4}+\beta_{5}\right) F^{(4)}(U(1, t)) \\
&-\left(\beta_{1} \alpha_{1}^{2}+\beta_{2} \alpha_{2}^{2}+\beta_{3} \alpha_{3}^{2}+\beta_{4} \alpha_{4}^{2}+\beta_{5} \alpha_{5}^{2}\right) F^{(3)}(U(1, t)) \\
&+\left(\beta_{1} \alpha_{1}^{4}+\beta_{2} \alpha_{2}^{4}+\beta_{3} \alpha_{3}^{4}+\beta_{4} \alpha_{4}^{4}+\beta_{5} \alpha_{5}^{4}\right) F^{(2)}(U(1, t)) \\
&-\left(\beta_{1} \alpha_{1}^{6}+\beta_{2} \alpha_{2}^{6}+\beta_{3} \alpha_{3}^{6}+\beta_{4} \alpha_{4}^{6}+\beta_{5} \alpha_{5}^{6}\right) F^{(1)}(U(1, t)) \\
&+\left(\beta_{1} \alpha_{1}^{8}+\beta_{2} \alpha_{2}^{8}+\beta_{3} \alpha_{3}^{8}+\beta_{4} \alpha_{4}^{8}+\beta_{5} \alpha_{5}^{8}\right) F(U(1, t))
\end{aligned}
$$

\subsection{Remarks}

The Runge-Kutta method has been applied to solve the integral equation resulting from the heat conduction problem with a combined convection and radiation. In particular, the nonlinear ordinary differential equation has been determined for both the third and the fifth order of approximation. As one may notice, this method is not very practical for calculating the temperature at small times. The reason is that a smaller time one takes, more terms will be needed for the infinite series to be represented correctly, which in turn may result in a high-ordered nonlinear ordinary differential equation with a huge expression. Thus, the method is usually used to compute the surface temperature at large times. In the following chapter, we will introduce another numerical method which has a different approach of finding the solution of the stated problem. It is called the finite difference method. The method is different from the previous numerical techniques in a sense that instead of solving the integral equation, it approximates the partial differential equation and the boundary conductions directly. Details will be found in the next chapter. 


\section{CHAPTER IV}

\section{The Finite Difference Method}

\subsection{Introduction}

The basic idea of the finite difference method is the transformation of a continuous model into a discrete system by replacing any continuous domain in the model with a denumerable domain. In applying this idea to differential equations, all the derivatives in the equation are simply replaced by finite difference approximations. Thus, the unknowns in the differential equation have a countable domain, and the resulting discrete system is solved numerically.

In the theory of numerical analysis, the significance of the computed solution of a finite difference scheme in relation to approximating the exact solution is dependent upon three elements. They are consistency, convergence, and stability. Consistency is a condition used to assure that as $\Delta \mathrm{x}$ approaches zero, the truncation errors of the scheme also go to zero. It implies that the finite difference can be an arbitrarily accurate approximation to the derivative by the partition refinement. For the convergence of an approximation, it assures that if $\Delta x$ goes to zero, the difference between the computed and the exact values also go to zero. In other words, any desired accuracy of the approximated solution can be achieved. The last element is the stability. The stability of a scheme concerns the growth of the errors found in the calculations which are needed to solve the 
system of linear equations. A scheme is claimed to be conditionally stable if the round-off error does not amplify as the time step goes under a certain value. In the Lax Equivalence Theorem, the relationships of these three conditions are stated. It tells that given a properly posed initial-value problem and a finite difference scheme which satisfies the consistency condition, stability is the necessary and sufficient condition for convergence. There are many difference approximations and methods of solving the discrete system that are available in numerical analysis. Different choices of approximation and methods of solving the system will lead to different accuracy in the approximation of the solutions. This chapter will only focus on a particular finite difference scheme used to approximate the governing partial differential equation in the stated problem and an algorithm for solving the discretized system.

\subsection{Crank-Nicolson Scheme}

Suppose $x$ lies between $x_{0}$ and $x_{f}$ and $t \geq t_{0}$. Let $\Delta x$ and $\Delta t$ be increments of $x$ and $t$, respectively. The $x-t$ space can be partitioned into a grid network in which the points are given by $x=x_{0}+j \Delta x$ and $t=t_{0}+n \Delta t$, where $j=0,1,2$, $\ldots, N$, with $\mathrm{N}$ be the number of nodes, and $\mathrm{n}=0,1,2, \ldots$. When $\Delta \mathrm{x}$ and $\Delta \mathrm{t}$ are constants, the mesh obtained is uniform, and the temperature at $x=x_{0}+i \Delta x$, written as $x_{j}$, and $t=t_{0}+n \Delta t$, written as $t_{n}$, is denoted by $U_{j}^{n}$.

As previously mentioned, there are several ways of choosing a finite difference operator for replacement of derivatives. If the average of the 
forward and backward difference schemes is used for the space discretization and the forward difference scheme is written about the point $x_{i}, t_{j+1 / 2}$ for time discretization, the governing partial differential equation becomes a second-order-accurate in both $x$ and $t$ finite difference equation. It is given by:

$$
\mathrm{U}_{\mathrm{j}-1}^{\mathrm{n}+1}+(-2-2 \beta) \mathrm{U}_{\mathrm{j}}^{\mathrm{n}+1}+\mathrm{U}_{\mathrm{j}+1}^{\mathrm{n}+1}=-\mathrm{U}_{\mathrm{j}-1}^{\mathrm{n}}+(2-2 \beta) \mathrm{U}_{\mathrm{j}}^{\mathrm{n}}-\mathrm{U}_{\mathrm{j}+1}^{\mathrm{n}}
$$

where

$$
\beta=\frac{\Delta x^{2}}{\Delta t}
$$

The above scheme is called the Crank-Nicolson equation. Since it is of second order, the truncation error associated with (4.1) is $\mathrm{O}\left(\Delta \mathrm{x}^{2}+\Delta \mathrm{t}^{2}\right)$. Notice that the temperature at time $t_{n+1}$ is a function of unknown and known temperatures at the points shown on the Fig. 4.1.

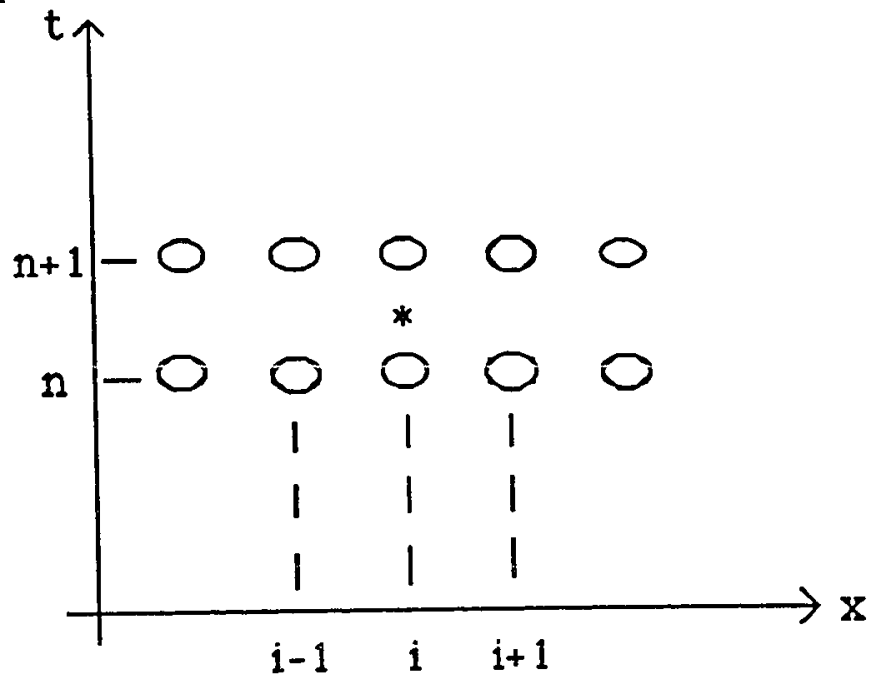

Fig. 4.1 Graphical representation for Crank-Nicolson Equation 
Though the Crank-Nicholson scheme is unconditionally stable in von Neumann sense [10], it is well known that the finite difference solution may suffer from severe oscillations when it is applied to the Robin's type boundary conditions with the choice of a time step which is large relative to the spatial step [10]. This phenomenon becomes more pronounced especially when the nonlinear boundary conditions are treated with a crude approximation where the surface temperatures are not determined with sufficient accuracy. Thus, in the problems considered, the Crank-Nicolson equation is only applied to the boundary at $x=0$ and the space between the boundaries, that is,

$$
\mathrm{j}=\left\{\begin{array}{l}
0, \ldots \mathrm{N}-1 \text { for case } 1 \\
1, \ldots, \mathrm{N}-1 \text { for case } 2
\end{array}\right.
$$

To ensure that the oscillation is eliminated, the implicit backward finite difference scheme which is satisfactory with all types of boundary conditions is adopted at the boundary, $x=1$. The equation at $x=1$ is given by:

$$
\mathrm{U}_{\mathrm{N}-1}^{\mathrm{n}+1}+(-2-\beta) \mathrm{U}_{\mathrm{N}}^{\mathrm{n}+1}+\mathrm{U}_{\mathrm{N}+1}^{\mathrm{n}+1}=-\beta \mathrm{U}_{\mathrm{N}}^{\mathrm{n}}
$$

where

$$
\beta=\frac{(\Delta x)^{2}}{\Delta t}
$$

There is a fictitious point outside the computational domian in (4.3), that is, the unknown temperature at $\mathrm{N}+1$ is denoted as $\mathrm{U}_{\mathrm{N}+1}^{\mathrm{n}+1}$. To eliminate that point, 
difference method to approximate the derivative in the radiative boundary condition, (1.2c) becomes

$$
\frac{\mathrm{U}_{\mathrm{N}+1}^{\mathrm{n}+1}-\mathrm{U}_{\mathrm{N}-1}^{\mathrm{n}+1}}{2 \Delta \mathrm{x}}-\alpha_{3} \mathrm{U}_{\mathrm{N}}^{\mathrm{n}+1}=\mathrm{F}\left(\mathrm{U}_{\mathrm{N}}^{\mathrm{n}+1}\right)
$$

where $F$ is the right-hand side of (1.2c). Algebraically manipulating (4.4) yields the following equation:

$$
\mathrm{U}_{\mathrm{N}+1}^{\mathrm{n}+1}=\mathrm{U}_{\mathrm{N}-1}^{\mathrm{n}+1}+2 \Delta \mathrm{x} \alpha_{3} \mathrm{U}_{\mathrm{N}}^{\mathrm{n}+1}+2 \Delta \mathrm{x} F\left(\mathrm{U}_{\mathrm{N}}^{\mathrm{n}+1}\right)
$$

Substituting (4.5) into (4.3), the resulting expression becomes

$$
2 U_{N-1}^{n+1}+\left(-2-\beta+2 \Delta x \alpha_{3}\right) U_{N}^{n+1}=-\beta U_{N}^{n}-2 \Delta x F\left(U_{N}^{n+1}\right)
$$

which is a nonlinear equation in $U_{\mathbb{N}}^{\mathfrak{n}+1}$.

Now, (4.1) and (4.6) constitute a set of simultaneous equations at each time step. In fact, in matrix representation, the resulting system is of the following form:

$$
\mathbf{A} \mathbf{U}=\mathbf{B}
$$

where $A$ is a iridiagonal matrix, $\mathrm{B}$ is a matrix of all the known values found in each equation, and $\mathbf{U}$ is a matrix of the unknown temperatures at each space node at a particular moment of time. So, for each time level, the transient temperature is given by the solutions of a system of equations.

The Thomas algorithm can be used to solve a tridiagonal system of linear equations. Clearly, except the last equation, all the equations in (4.7) are linear. As given in the appendix, the first half of the algorithm can be directly applied to the system except for the case where $i=N$. In the case of $i=$ 58 
directly applied to the system except for the case where $i=N$. In the case of $i=$ $N$, substituting $d(N)$ in the first Do-loop in the expression right after the first loop yields

$$
\begin{aligned}
& U_{N}^{n+1}=\frac{d(N)-\text { ratio*d(N-1) }}{b(N)} \\
\Rightarrow \quad & \quad U_{N}^{n+1}=\frac{-\beta U_{N}^{n}-2 \Delta x F\left(U_{N}^{n+1}\right)-\text { ratio } * d(N-1)}{b(N)}
\end{aligned}
$$

with $b(\mathrm{~N}), d(\mathrm{~N}-1)$, and ratio computed in the first DO-Loop. Now, (4.9) can be rewritten as

$$
\frac{-\beta U_{N}^{n}-2 \Delta x F\left(U_{N}^{n+1}\right)-\text { ratio } * d(N-1)}{b(N)}-U_{N}^{n+1}=0
$$

Letting $f$ be the left-hand side of (4.10), (4.10) becomes

$$
f\left(U_{N}^{n+1}\right)=0
$$

Thus, the surface temperature at a new time is given by the solution of the nonlinear equation (4.11). There are many existing numericai methods to tackle such an equation with confidence. For instance, the Newton-Raphson method. Once the surface temperature is determined, the second half of the algorithm can be used to compute the solutions.

In the following, two specific cases, namely, the flat plate and the sphere will be considered. The tridiagonal system of each case will be obtained. 


\subsection{Two Special Cases}

\subsubsection{The Flat Plate}

The parameters corresponding to this case can be found in chapter 1 and will not be given here again. Applying the finite difference method outlined above to the governing equations leads to the following:

Consider the Crank-Nicholson scheme for $\mathrm{j}=0, \ldots, \mathrm{N}-1$. For $\mathrm{j}=0$,

$$
\mathrm{U}_{-1}^{\mathrm{n}+1}+(-2-2 \beta) \mathrm{U}_{0}^{\mathrm{n}+1}+\mathrm{U}_{1}^{\mathrm{n}+1}=-\mathrm{U}_{-1}^{\mathrm{n}}+(2-2 \beta) \mathrm{U}_{0}^{\mathrm{n}}-\mathrm{U}_{1}^{\mathrm{n}} \text {. }
$$

To eliminate the fictitious points, the boundary condition at $x=0$ in discretized form is given by

$$
\frac{\mathrm{U}_{1}^{\mathrm{n}+1}-\mathrm{U}_{-1}^{\mathrm{n}+1}}{2 \Delta \mathrm{x}}=0
$$

Thus,

$$
\mathrm{U}_{1}^{\mathrm{n}+1}=\mathrm{U}_{-1}^{\mathrm{n}+1}
$$

and

$$
\mathrm{U}_{1}^{\mathrm{n}}=\mathrm{U}_{-1}^{\mathrm{n}}
$$

Substituting (4.14) and (4.15) in (4.12) produces

$$
(-2-2 \beta) U_{0}^{n+1}+2 U_{1}^{n+1}=(2-2 \beta) U_{0}^{n}-2 U_{1}^{n}
$$

For $j=1, \ldots, N-1$,

$$
\begin{gathered}
\mathrm{U}_{0}^{\mathrm{n}+1}+(-2-2 \beta) \mathrm{U}_{1}^{\mathrm{n}+1}+\mathrm{U}_{2}^{\mathrm{n}+1}=-\mathrm{U}_{0}^{\mathrm{n}}+(2-2 \beta) \mathrm{U}_{1}^{\mathrm{n}}-\mathrm{U}_{2}^{\mathrm{n}} \\
\vdots \\
\vdots \\
\mathrm{U}_{\mathrm{N}-2}^{\mathrm{n}+1}+(-2-2 \beta) \mathrm{U}_{\mathrm{N}-1}^{\mathrm{n}+1}+\mathrm{U}_{\mathrm{N}}^{\mathrm{n}+1}=-\mathrm{U}_{\mathrm{N}-2}^{\mathrm{n}}+(2-2 \beta) \mathrm{U}_{\mathrm{N}-1}^{\mathrm{n}}-\mathrm{U}_{\mathrm{N}}^{\mathrm{n}}
\end{gathered}
$$

When $\mathrm{j}=\mathrm{N}$, as shown before, the equation becomes

$$
2 U_{N-1}^{n+1}+\left(-2-\beta+2 \Delta x \alpha_{3}\right) U_{N}^{n+1}=-\beta U_{N}^{n}-2 \Delta x F\left(U_{N}^{n+1}\right)
$$

In matrix representation, with initial values $U_{j}^{0}=1$, where $j=0, \ldots, N$., 


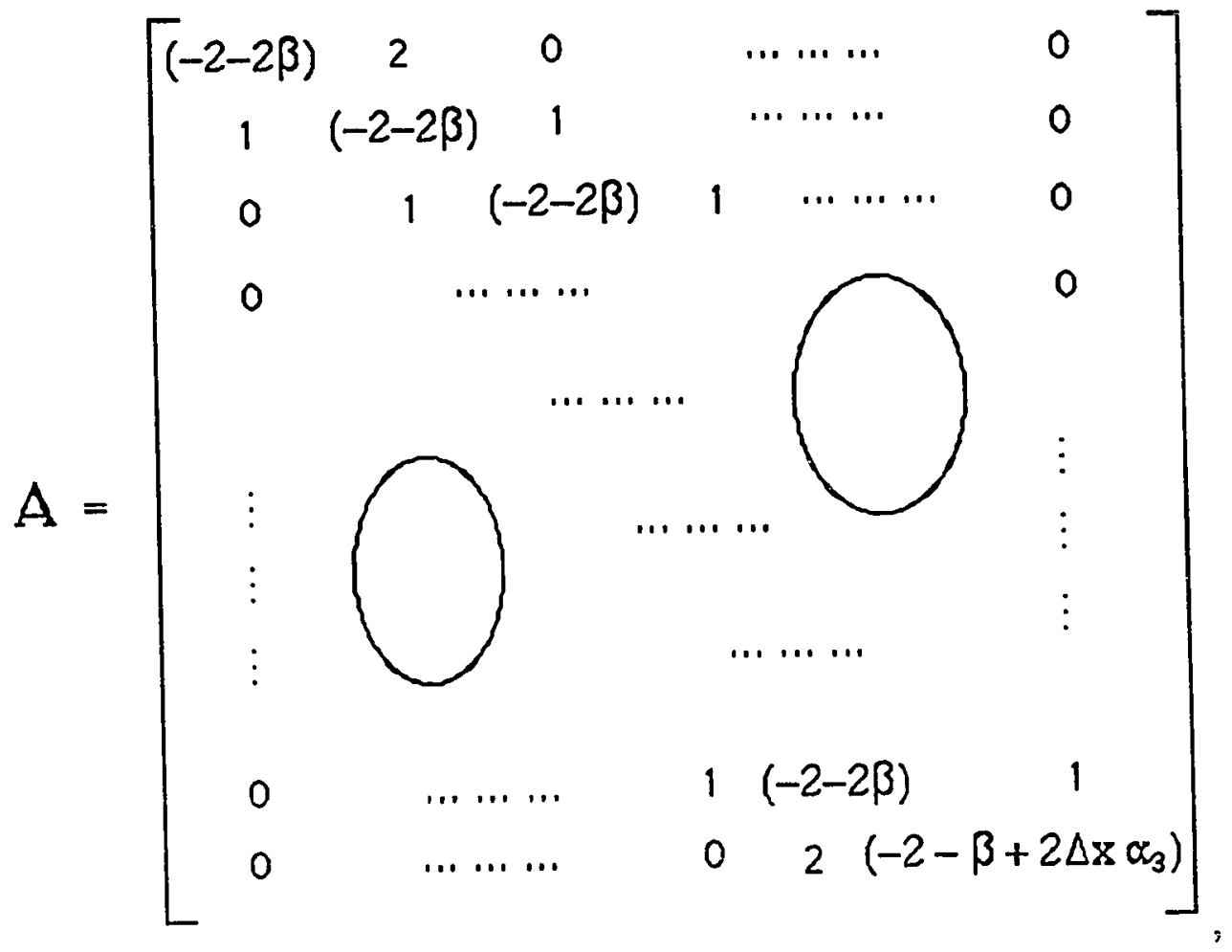




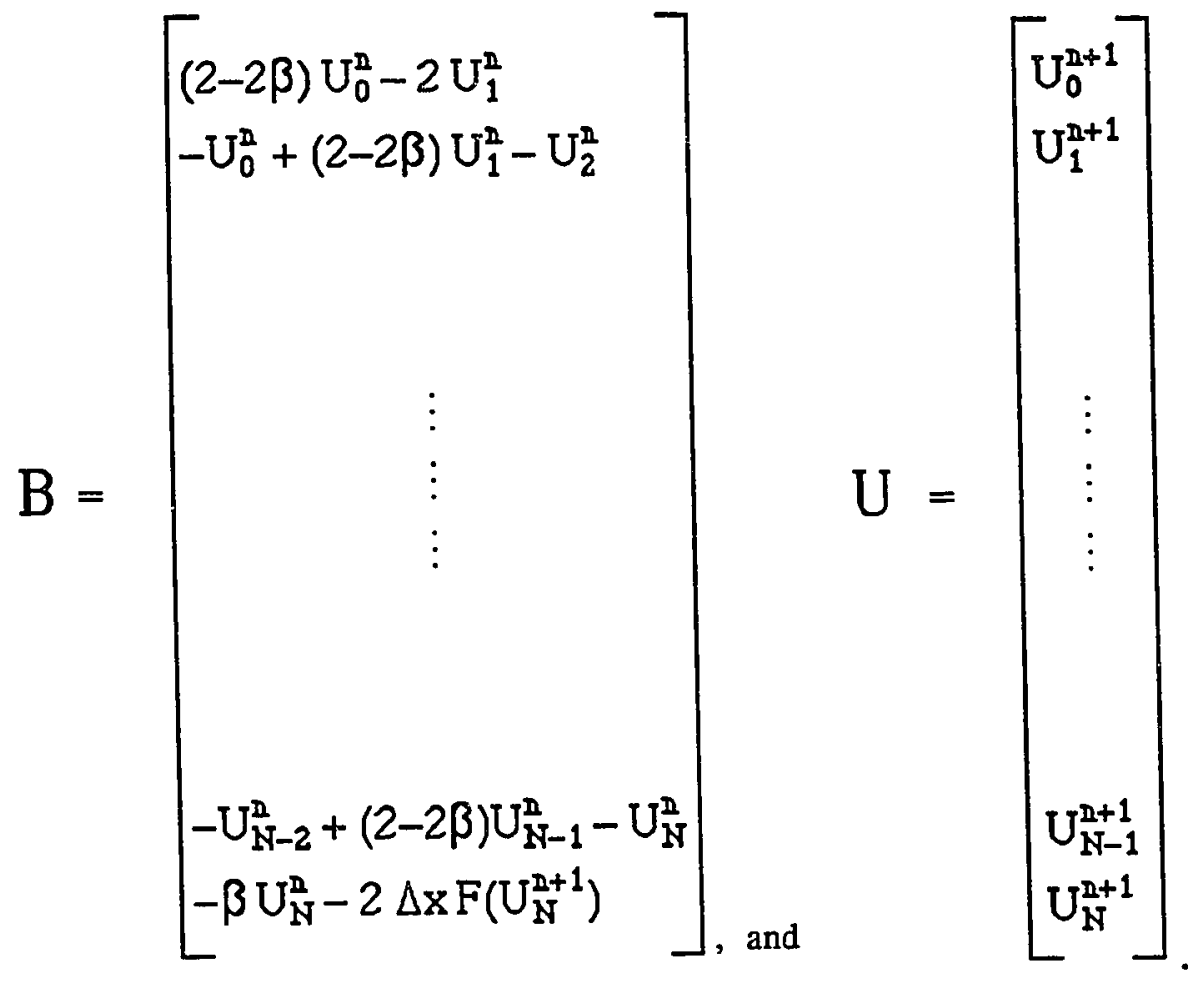

\subsubsection{The Sphere}

The parameters again can be obtained as in chapter 1 and will not be given here. Applying the finite difference method outlined above to the governing equations leads to the following:

Consider the Crank-Nicholson for $j=1, \ldots, N-1$. For $j=1$,

$$
\mathrm{U}_{0}^{\mathrm{n}+1}+(-2-2 \beta) \mathrm{U}_{1}^{\mathrm{n}+1}+\mathrm{U}_{2}^{\mathrm{n}+1}=-\mathrm{U}_{0}^{\mathrm{n}}+(2-2 \beta) \mathrm{U}_{1}^{\mathrm{n}}-\mathrm{U}_{2}^{\mathrm{n}} \text {. }
$$

However,

$$
U_{0}^{n+1}=0 \text { and } U_{0}^{n}=0
$$

Substituting (4.22) in (4.21) produces 


$$
(-2-2 \beta) U_{1}^{n+1}+U_{2}^{n+1}=(2-2 \beta) U_{1}^{n}-U_{2}^{n} .
$$

For $\mathrm{j}=2, \ldots, \mathrm{N}-1$,

$$
\begin{gathered}
\mathrm{U}_{1}^{\mathrm{n}+1}+(-2-2 \beta) \mathrm{U}_{2}^{\mathrm{n}+1}+\mathrm{U}_{3}^{\mathrm{n}+1}=-\mathrm{U}_{1}^{\mathrm{n}}+(2-2 \beta) \mathrm{U}_{2}^{\mathrm{n}}-\mathrm{U}_{3}^{\mathrm{n}}, \\
\vdots \\
\vdots \\
\mathrm{U}_{\mathrm{N}-2}^{\mathrm{n}+1}+(-2-2 \beta) \mathrm{U}_{\mathrm{N}-1}^{\mathrm{n}+1}+\mathrm{U}_{\mathrm{N}}^{\mathrm{n}+1}=-\mathrm{U}_{\mathrm{N}-2}^{\mathrm{n}}+(2-2 \beta) \mathrm{U}_{\mathrm{N}-1}^{\mathrm{n}}-\mathrm{U}_{\mathrm{N}}^{\mathrm{n}}
\end{gathered}
$$

When $\mathbf{j}=\mathrm{N}$, as shown before, the backward scheme is used, thus,

$$
2 U_{N-1}^{n+1}+\left(-2-\beta+2 \Delta x \alpha_{3}\right) U_{N}^{n+1}=-\beta U_{N}^{n}-2 \Delta x F\left(U_{N}^{n+1}\right) \text {. }
$$

In matrix representation, with initial values $U_{j}^{0}=j \Delta x$, where $j=1, \ldots, N$,

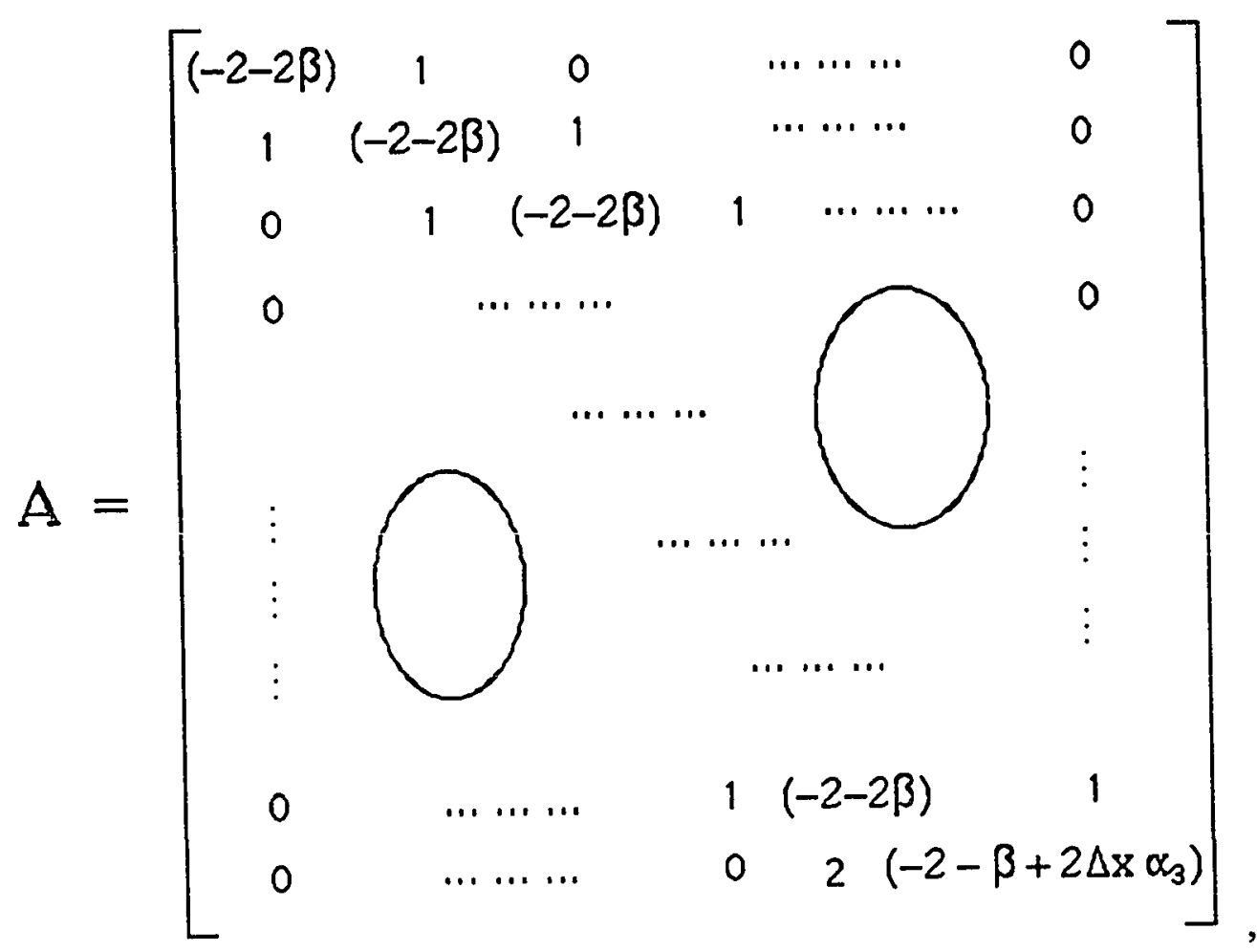



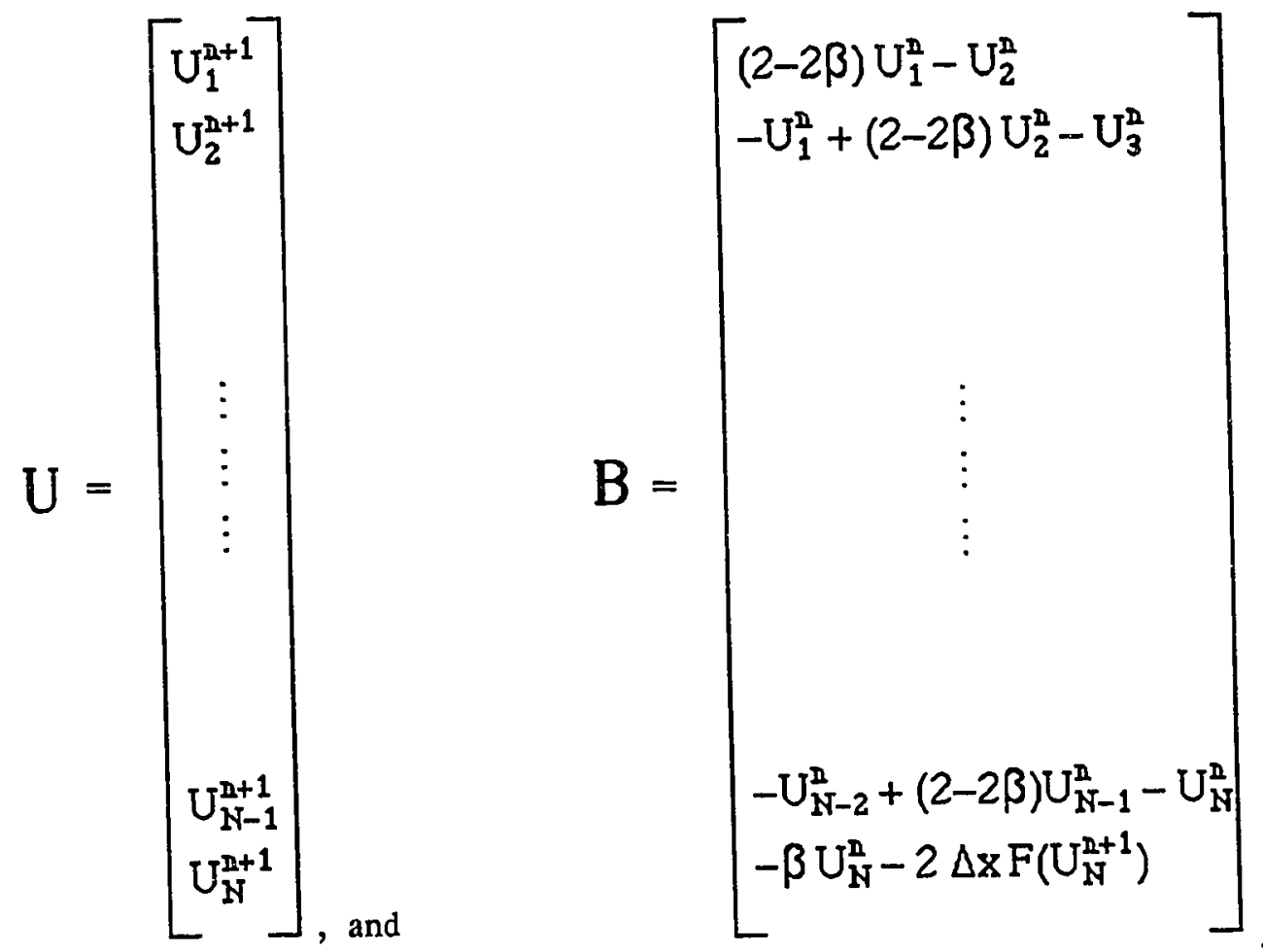

\subsection{Stability}

Even though the backward analog is implemented on the radiative boundary, according to the numerical experiments, the method still suffers from the oscillation when a large time step is imposed. So far, not a single formula has been developed for the stability criteria of an implicit finite difference scheme with nonlinear boundary conditions. However, two stability formulas of the related problems, which are found in the literature, can serve as a guildline in choosing the time step for the problems considered. The first one is according to Lawson and Morris [7]. They deduce the stability criteria for the Crank-Nicolson equation with linear boundary conditions as 


$$
\Delta \mathrm{t}<\frac{2 \Delta \mathrm{x}}{\pi}
$$

Another stability criterion is due to Milton and Goss [7] who applied the laws of thermodynamics in developing the stability requirement for an explicit finite difference scheme with nonlinear boundary conditions. It turned out that the time step required for the stability is restricted by:

$$
\Delta t \leq \frac{(\Delta x)^{2}\left\{U_{N}^{n} \mid \max -U_{N}^{n}\right\}}{2\left\{U_{N-1}^{n}-U_{N}^{n}-\Delta x\left(U_{N}^{n}\right)^{4}-\alpha_{3} \Delta x U_{N}^{n}\right\}},
$$

where the maximum of $\mathrm{U}_{\mathrm{N}}^{\mathrm{n}}$ can be found by setting the following equation

$$
\frac{\Delta U_{N}}{\Delta t}=A-B U_{N}-C U_{N}^{4}
$$

equal to zero, where

$$
\begin{aligned}
& \Delta \mathrm{U}_{\mathrm{N}}=\mathrm{U}_{\mathrm{N}}^{\mathrm{n}+1}-\mathrm{U}_{\mathrm{N}}^{\mathrm{n}}, \\
& \mathrm{A}=\frac{2 \mathrm{U}_{\mathrm{N}-1}^{\mathrm{n}}}{(\Delta \mathrm{x})^{2}}, \\
& \mathrm{~B}=\frac{2[\Delta \mathrm{x}-1]}{(\Delta \mathrm{x})^{2}}, \\
& \mathrm{C}=\frac{2 \Delta \mathrm{x}}{(\Delta \mathrm{x})^{2}} .
\end{aligned}
$$

Because this formula is not very practical in use, (4.28) will be chosen as a guildline for selecting the time step of the method. 


\subsection{Refinement of a Partition and Extrapolation Techniques}

The solution of the partition of the domain covered has a great influence on the accuracy of the solution obtained. The choice of grid points is determined by knowledge of the problem and by numerical experimentation. Here, two ways of improving the accuracy of the finite difference method are presented, and one of the two was chosen to be implemented in the numerical results.

One way to improve the accuracy is to first solve the problem using one spacing and then refine the partition (usually made smaller) and then repeat the computation. If the comparison shows large differences, the process is repeated for smaller and smaller grid sizes until a desired accuracy is achieved. This method may result in a prolonged computational time for the solution.

The second way is called extrapolation. The simple ingenious idea of the technique, which seems to date back to Richardson in 1910, is the following: one solves the same type of problem over a prescribed interval, for example $[0,1]$, several times with successively smaller stepsizes. Thus, one obtains a sequence of approximations

$$
\gamma\left(1, h_{0}\right), \gamma\left(1, h_{1}\right), \ldots .
$$

for a given sequence of stepsizes

$$
h_{0}>h_{1}>\cdots>0 \text {. }
$$

The successive stepsizes $h_{i}$ can be defined in terms of the input stepsize $h$ by

$$
h_{i}=\frac{h}{n_{i}}, i=0,1,2, \ldots \text {. }
$$


Thus, any stepsize sequence $\left\{h_{i}\right\}$ can be characterized by the associated integer sequence $\left\{n_{i}\right\}$. Following are some examples of integer sequences:

$$
\begin{aligned}
& \{1,2,4,8,16,32, \ldots\} \quad \text { (Romberg sequence), } \\
& \{1,2,4,6,8,12, \ldots\} \quad \text { (Bulirsch sequence), } \\
& \{1,2,3,4, \ldots\} \quad \text { (harmonic sequence). }
\end{aligned}
$$

So, the numerical solution at $x$ is computed for a sequence of stepsizes $h_{i}$ and denoted by $\mathrm{T}_{\mathrm{i}, 0}:=\gamma\left(1, \mathrm{~h}_{\mathrm{i}}\right)$. Then, the extrapolation tableau,

$$
\begin{aligned}
& \mathrm{T}_{00} \\
& \mathrm{~T}_{10} \mathrm{~T}_{11} \\
& \mathrm{~T}_{20} \mathrm{~T}_{21} \mathrm{~T}_{22},
\end{aligned}
$$

is calculated for $x$ according to two types of commonly used extrapolation schemes :

a) Aitken-Neville algorithm

$$
\begin{aligned}
& \text { For } i=1,2, \ldots \text { and } k=1,2,3, \ldots, i \\
& T_{i, k}=T_{i, k-1}+\frac{T_{i, k-1}-T_{i-1, k-1}}{\left(\frac{n_{i}}{n_{i-k}}\right)^{2}-1}
\end{aligned}
$$

b) Rotational extrapolation

$$
\begin{aligned}
& \text { For } i=1,2, \ldots \text { and } k=1,2,3, \ldots, i \\
& T_{i,-1}=0, \\
& T_{i, k}=T_{i, k-1}+\frac{T_{i, k-1}-T_{i-1, k-1}}{\left(\frac{n_{i}}{n_{i-k}}\right)^{2}\left[1-\frac{T_{i, k-1}-T_{i-1, k-1}}{T_{i, k-1}-T_{i-1, k-2}}\right]-1}
\end{aligned}
$$


In this study, extrapolation scheme (4.32) with the Romberg sequence will be implemented when the finite difference method is used to solve the numerical solution of the stated problems. However, that would excessively increase the amount of computational time. 


\section{CHAPTER V}

\section{Numerical Results}

\subsection{Introduction}

The problem described in section 0.2 was solved numerically for two special cases, namely, the flat plate $\left(\alpha_{1}=1, \alpha_{2}=0, \alpha_{3}=-1, h=1\right)$ and the sphere $\left(\alpha_{1}=0, \alpha_{2}=-1, \alpha_{3}=1, h=1\right)$. Since a lot of numerical results of the problem computed by the successive approximations method are available in some of the papers $[3,4]$, in this thesis, only the Runge-Kutta method and the finite difference method are applied to the problem for study. Programs were written in Fortran 77 and were set up in such a way that a time step can be input. This feature enables one to approximate the maximum time step that can be used in a particular numerical method. All calculations were done using double precision arithmetic yielding 12-digit accuracy. Numerical results generated by the methods are compared and discussed.

The Runge-Kutta and the finite difference methods were implemented to solve both cases. In particular, three different order approximations of the Runge-Kutta method were programmed to solve the integral equations derived by the Laplace transform method. Inefficiency of a high order Runge-Kutta method motivates the use of the finite difference technique. Again, the method was implemented in both cases for various time steps. An extrapolation algorithm was used to optimize the accuracy of the solutions. Some of the numerical results are tabulated and plotted in such a way that a 
comparsion can be made. Notice that the Runge-Kutta method was not applied to the integral equations obtained by eigenvalue expansion method. The reason is that the lag parts of those integral equations diverge when time is equal to zero, and thus, the initial values of the nonlinear ordinary differential equations cannot be computed.

\subsection{Results for the flate plate and the Sphere}

Integral equations (1.39) and (1.43) were solved using the Runge-Kutta method of orders 1, 3, and 5. The first order approximation can be found in [5], whereas the third and the fifth order approximations are described in sections 3.3 and 3.4 , respectively. Solutions of the nonlinear ordinary differential equations corresponding to (1.39) and (1.43) were obtained using the four-order Runge-Kutta method developed by Zurmuhl [15]. The results show that solutions of a high order approximation fall below those of a lower order approximation (Fig. 5.1, Fig. 5.2, Fig. 5.3).

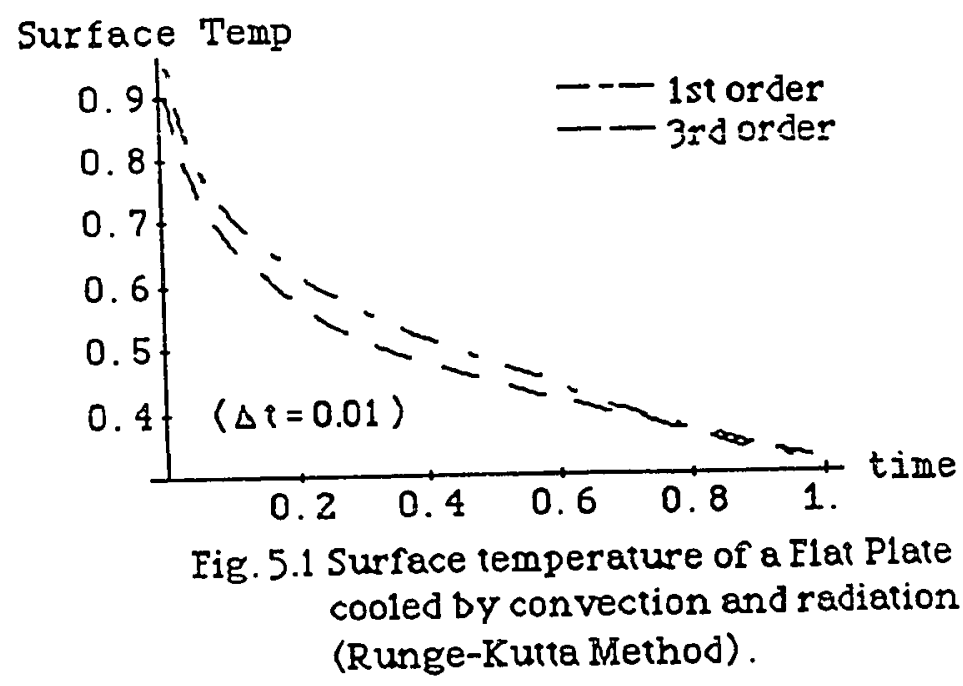




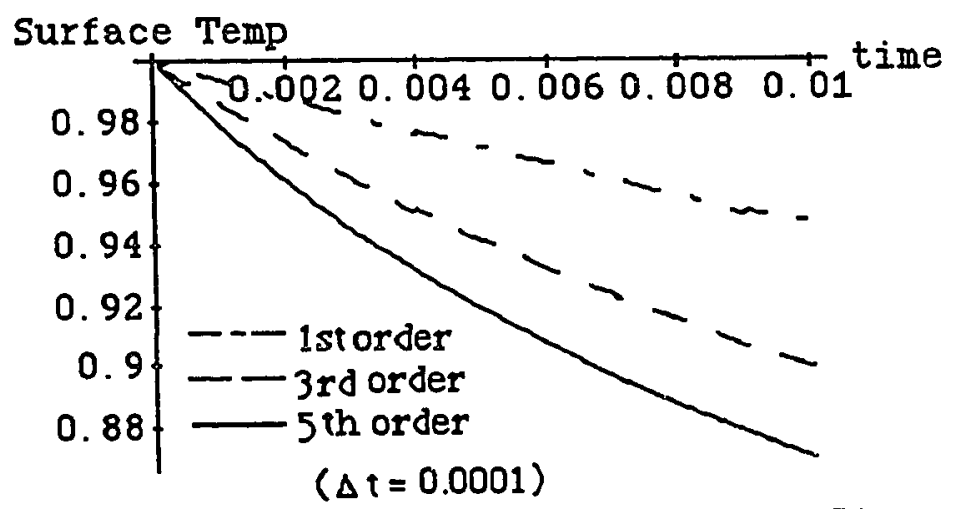

Fig. 5.2 Surface temperature of a Flat Plate cooled by convection and radiation (Runge-Kutta Method).

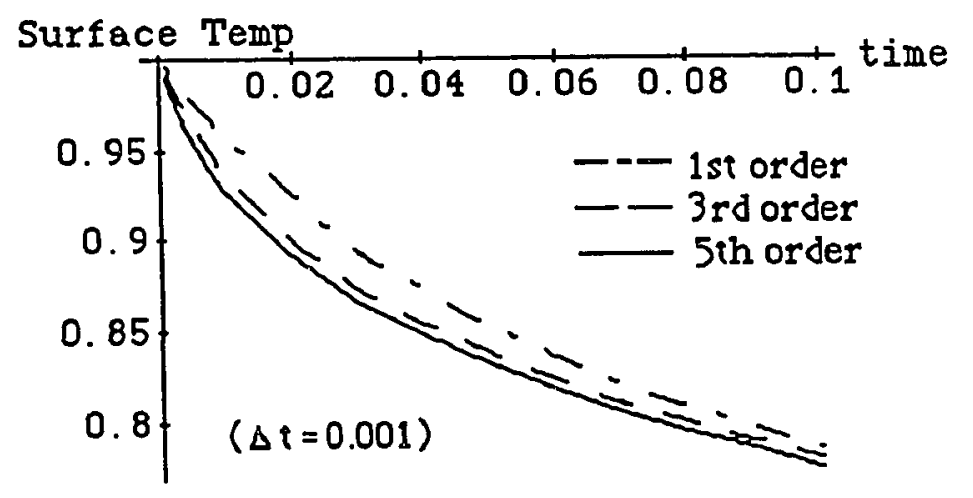

Fig. 5.3 Surface temperaiure or a sphere cooled by convection and radiation (Runge-Kutta Method).

With respect to time step, we do not have the same phenomenon as in the order of approximation. In a fixed order approximation method, the solution curves for a smaller time step fall below those for a larger time step at small times (approx. less than 0.2) and above at large times (Fig. 5.4). 


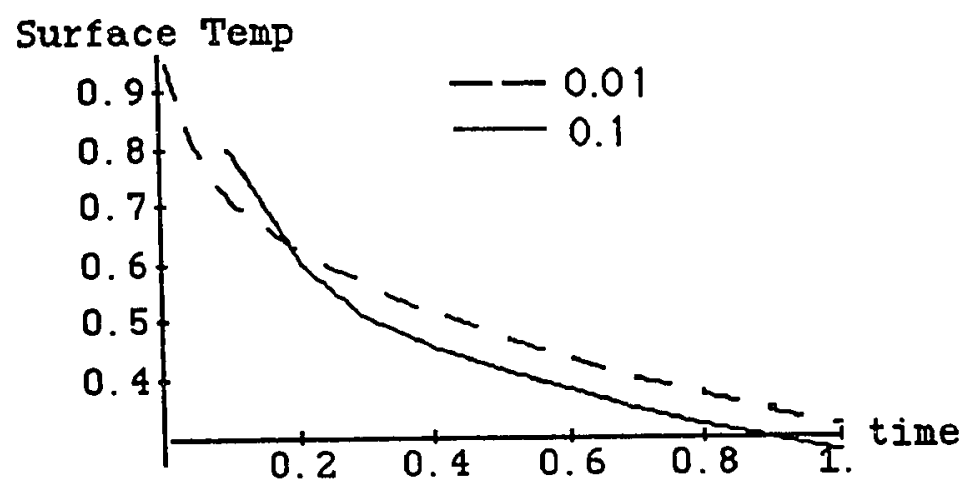

Fig. 5.4 Surface temperature of a Flat Plate cooled by convection and radiation (Runge-Kutta Method of the First Order)

According to numerical experiments, the stability requirements for the Runge-Kutta method of orders 1,3 , and 5 are approximately $0.1,0.01$, and 0.001 , respectively. As mentioned, a drawback of the Runge-Kutta method is that it requires the solution of a heuristic nonlinear ordinary differential equation for a high order approximation. This led to an attempt to use an easier algorithm, and for this reason, the finite difference method was implemented.

Equations (1.1) and $(1.2 \mathrm{a}, \mathrm{b}, \mathrm{c})$ were solved for the flat plate and for the sphere. The extrapolation formula used to improve the accuracy of the solutions is Aitken-Neville algorithm which is stated in equation (4.32). The results for various time steps are presented in tables 1 and 2 , and some of these results are plotted in Figs. 5.5 and 5.6. As tables 1 and 2 show, the situation where solution curves for a smaller time step fall below those for a larger time step holds in the finite difference method. 
Table 1

The Finite Difference Method for Various Time Steps

\begin{tabular}{l|l|c|c|}
\hline \multirow{2}{*}{ Time } & \multicolumn{3}{|c|}{$\Delta \mathbf{t}$} \\
\cline { 2 - 4 } & $1 \mathbf{0}^{2}$ & $1 \mathbf{0}^{\mathbf{3}}$ & $1 \mathbf{0}^{\mathbf{4}}$ \\
\hline 0.01 & 0.849395 & 0.843059 & 0.842539 \\
0.02 & 0.797214 & 0.793829 & 0.793609 \\
0.03 & 0.764701 & 0.762365 & 0.762256 \\
0.04 & 0.740419 & 0.738609 & 0.738559 \\
0.05 & 0.720827 & 0.719340 & 0.719328 \\
0.06 & 0.704316 & 0.703048 & 0.703063 \\
0.07 & 0.689998 & 0.688893 & 0.688927 \\
0.08 & 0.677333 & 0.676351 & 0.676400 \\
0.09 & 0.659595 & 0.665075 & 0.665137 \\
0.10 & 0.655626 & 0.654821 & 0.654893 \\
0.20 & 0.583564 & 0.583127 & \\
0.30 & 0.535891 & 0.535564 & \\
0.40 & 0.496573 & 0.496292 & \\
0.50 & 0.461310 & 0.461056 & \\
0.60 & 0.428810 & 0.428575 & \\
0.70 & 0.398614 & 0.398394 & \\
0.80 & 0.370501 & 0.370296 & \\
0.90 & 0.344321 & 0.344130 & \\
1.00 & 0.319948 & 0.319770 & \\
& & & \\
\hline
\end{tabular}

The Surface Temperature of a Flat Plate cooled by Convection and Radiation.

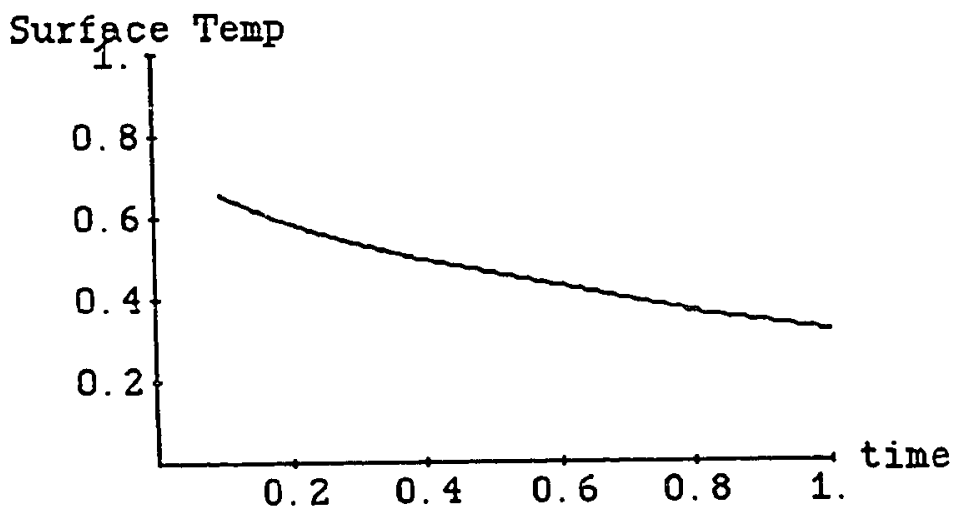

Fig. 5.5 Surface temperature of a flat plate cooled by convection and radiation (Einite Difference Method, $\Delta t=0.01$ ) 
Table 2

The Finite Difference Method for Various Time Steps

\begin{tabular}{l|l|l|l|}
\hline \multirow{2}{*}{ Time } & \multicolumn{3}{|c|}{$\Delta \mathrm{t}$} \\
\cline { 2 - 4 } & $10^{2}$ & $10^{3}$ & $10^{-4}$ \\
\hline 0.01 & 0.316249 & 0.912694 & 0.913001 \\
0.02 & 0.882656 & 0.880526 & \\
0.03 & 0.860270 & 0.858708 & \\
0.04 & 0.842779 & 0.841514 & \\
0.05 & 0.828134 & 0.827055 & \\
0.06 & 0.815380 & 0.814431 & \\
0.07 & 0.803988 & 0.803134 & \\
0.08 & 0.793626 & 0.792846 & \\
0.09 & 0.784075 & 0.774505 & \\
0.10 & 0.775178 & & \\
0.20 & 0.706513 & & \\
0.30 & 0.656561 & & \\
0.40 & 0.616906 & & \\
0.50 & 0.584412 & & \\
0.60 & 0.557215 & & \\
0.70 & 0.534052 & & \\
0.80 & 0.514033 & & \\
0.90 & 0.496514 & & \\
1.00 & 0.481017 & & \\
& & & \\
\hline
\end{tabular}

The Surface Temperature of a sphere cooled by Convection and Radiation.

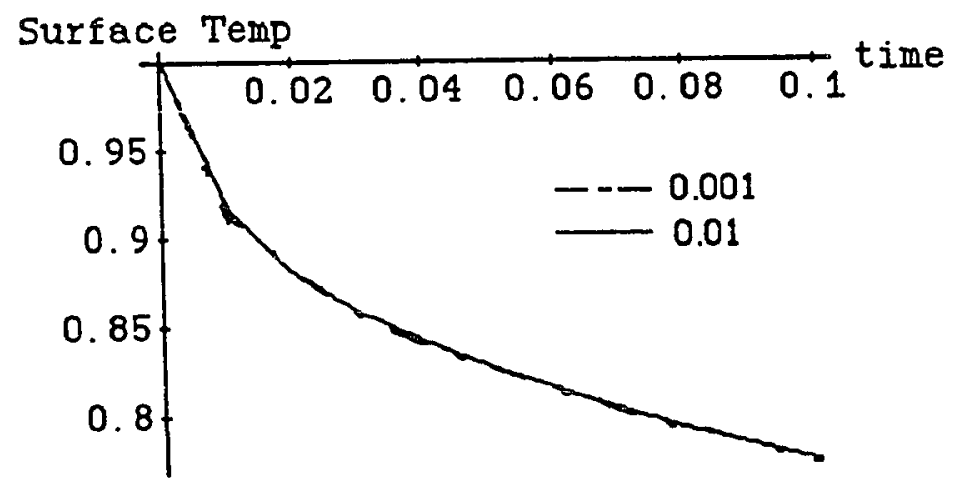

Fig. 5.6 Surface temperature of a sphere cooled by convection and radiation (Einite Difference Method). 
Even though the implicit scheme was implemented on the boundary, according to the numerical experiments, the solutions still exhibited oscillation when a large time step was chosen $(>0.01)$. This constraint of time step leads to large computational times for large time solutions. In addition, the extrapolation algorithm introduced in the program takes an enormous amount of computational time.

In Figs. 5.7 and 5.8, representative results by the Runge-Kutta and finite difference methods are presented for cooling. Tables 3 and 4 show that, when $\Delta t=0.01$ was used, the results obtained using the Runge-Kutta methed of orders 2 and 3 compared favorably with those obtained using the finite difference method. The difference in solution between the Runge-Kutta and the finite difference method in solution was less than $3.1 \%$ (relative error) in average for each case.

Table 3

Comparsion of the Runge-Kutta and the Finite Difference Methods $(\Delta t=0.01)$

\begin{tabular}{|c|c|c|c|}
\hline Time & 1st Order & 3rd Order & $\begin{array}{c}\text { Finite } \\
\text { Diff. }\end{array}$ \\
\hline $\begin{array}{l}0.10 \\
0.20 \\
0.30 \\
0.40 \\
0.50 \\
0.60 \\
0.70 \\
0.80 \\
0.90 \\
1.00\end{array}$ & $\begin{array}{l}0.712925 \\
0.619477 \\
0.562454 \\
0.516209 \\
0.475091 \\
0.437583 \\
0.403124 \\
0.371405 \\
0.342189 \\
0.315274\end{array}$ & $\begin{array}{l}0.667017 \\
0.567586 \\
0.514915 \\
0.477480 \\
0.445843 \\
0.417183 \\
0.390595 \\
0.365742 \\
0.342459 \\
0.320636\end{array}$ & $\begin{array}{l}0.655626 \\
0.583564 \\
0.535891 \\
0.496573 \\
0.461310 \\
0.428810 \\
0.398614 \\
0.370501 \\
0.344321 \\
0.319948\end{array}$ \\
\hline
\end{tabular}

The Surface Temperature of a Flat Plate cooled by Convection and Radiation. 


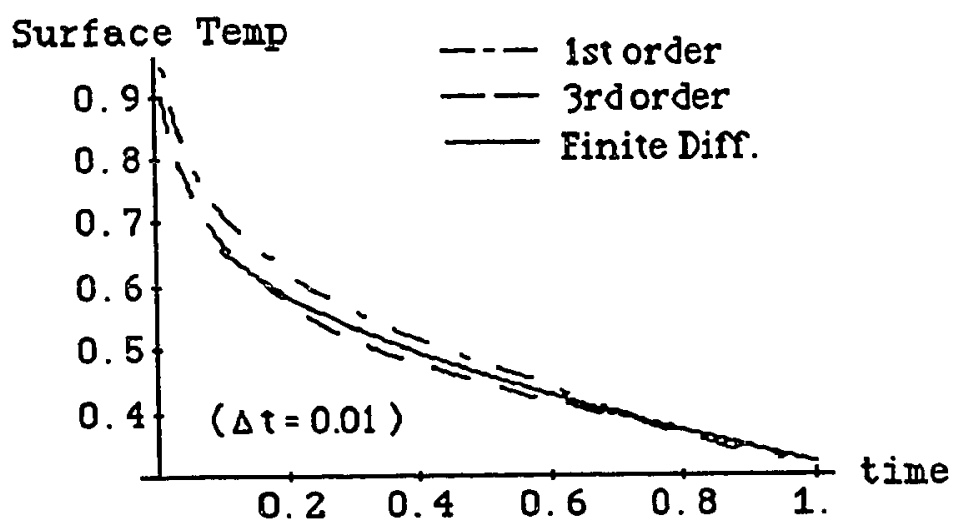

Fig. 5.7Comparsion of results for cooling a llat plate.

Table 4

Comparsion of the Runge-Kutta and the Finite Difference Methods $(\Delta t=0.01)$

\begin{tabular}{|c|c|c|c|}
\hline Time & 1st Order & 3rd Order & $\begin{array}{c}\text { Finite } \\
\text { Diff. }\end{array}$ \\
\hline $\begin{array}{l}0.10 \\
0.20 \\
0.30 \\
0.40 \\
0.50 \\
0.60 \\
0.70 \\
0.80 \\
0.90 \\
1.00\end{array}$ & $\begin{array}{l}0.785102 \\
0.705908 \\
0.651471 \\
0.609637 \\
0.576138 \\
0.548557 \\
0.525344 \\
0.505453 \\
0.488156 \\
0.472926\end{array}$ & $\begin{array}{l}0.754306 \\
0.665482 \\
0.622430 \\
0.591087 \\
0.565188 \\
0.542988 \\
0.523640 \\
0.506582 \\
0.491399 \\
0.477772\end{array}$ & $\begin{array}{l}0.775178 \\
0.706513 \\
0.656562 \\
0.616906 \\
0.584412 \\
0.557215 \\
0.534052 \\
0.514033 \\
0.496514 \\
0.481017\end{array}$ \\
\hline
\end{tabular}

The Surface Temperature of a Sphere cooled by Convection and Radiation. 


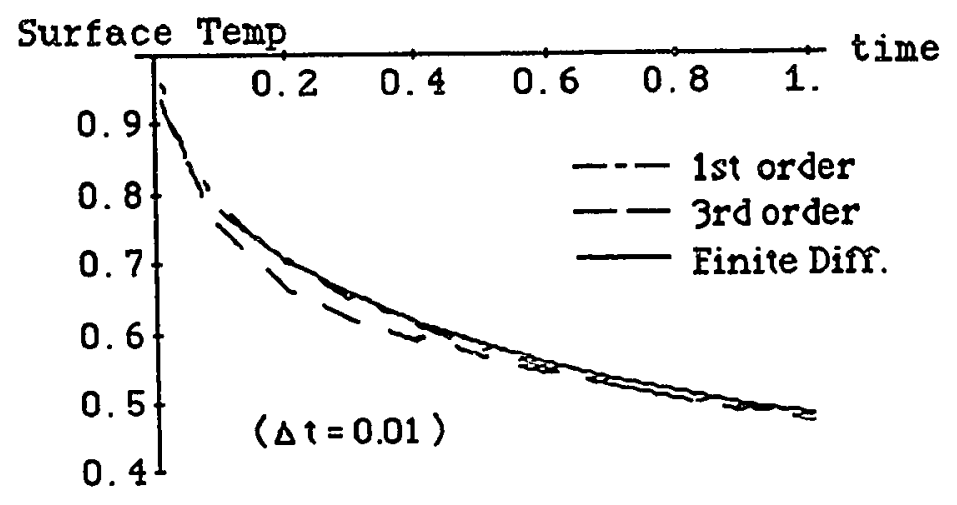

Fig. 5.8 Comparsion of results for cooling of a sphere.

Lastly, in Figs. 5.9 and 5.10, the results of two special cases solved by finite difference method are compared. The graph shows that, when $\Delta t=0.01$ was used, the surface temperature of a flate plate fell much faster than that of a sphere. This is probably due to the effect of the boundary condition at $x=0$ and the difference in the coefficient of the convective term.

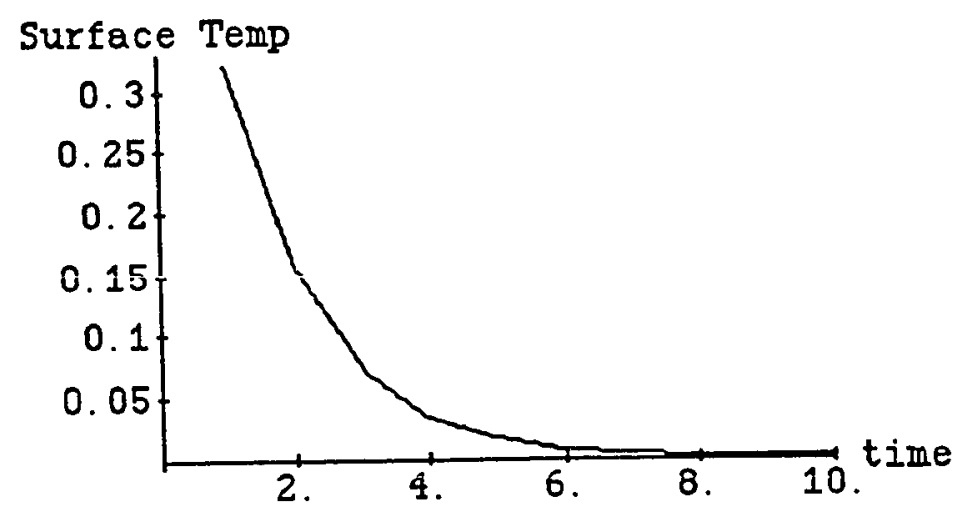

Fig. 5.9 Surface iemperature of a Ilat plate cooled by convection and radiation (Finite Difference Method, $\Delta t=0.01$ ) 


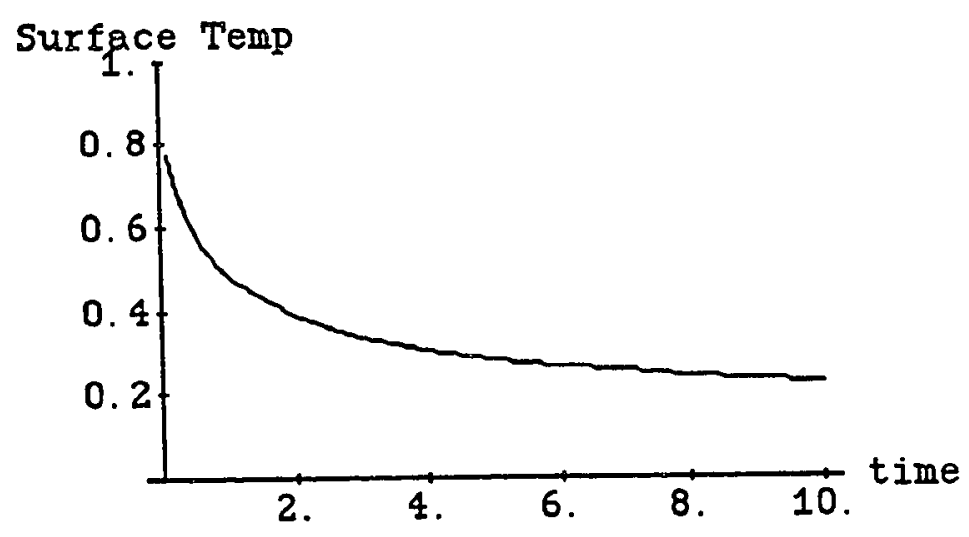

Fig. 5.10 Surface temperature of a sphere cooled by convection and radiation (Finite Differen $\approx e$ Method, $\Delta t=0.01$ ) 


\section{CHAPTER VI}

\section{CONCLUSION}

The study of the one-dimensional heat equation subjected to combined convective and radiative boundary conditions in rectangular coordinates was motivated by the advent of space technology where knowledge of the temperature of bodies in deep space is necessary, for instance, in the design of space shuttles.

The solids are assumed to be homogeneous, isotropic, and opaque to thermal radiation and to have temperature independent physical properties. This assumption leads to a linear heat equation. What determines the difficulty of the problem is the condition that prescribes the boundaries. According to the laws of physics, the heat flux of the radiative heat transfer is proportional to the fourth power of the temperature which causes nonlinearity at the boundaries.

Problems of this type were first solved using analytic techniques. The method that was chosen to obtain these solutions was the technique of integral transform. In particular, Laplace transform and eigenvalue expansion were used. The solutions which were explicitly determined at the surface for two special cases, namely, the flat plate and the sphere, are singular nonlinear Volterra integral equations of the second kind. Although they are not practical in determining the temperature at a particular time, 
these integral equations can help to deduce some useful information about the behavior of the surface temperature.

Since the analytic solutions found for the problem are not practical to use, numerical techniques are considered as an alternative. Two numerical schemes that are used to deal with the resulting integral equation are the Runge-Kutta method and the successive approximations method. Both techniques, which have been applied to a similar problem elsewhere, were developed for the integral equation in great detail. Conditions for the numerical solution to accurately approximate the exact solution and limitions of these schemes were discussed.

Another numerical technique which is directly applied to the governing equations was presented as a possible alternative to the numerical methods previously discussed. It is called the finite difference method in which the Crank-Nickolson scheme, the backward implicit scheme, and the Newton-Raphson method are combined to solve the surface temperature.

The Runge-Kutta methods of orders 1, 3, and 5 were programmed for (1.39) and (1.43), which are the integral equations corresponding to the flat plate and the sphere, respectively. The numerical results were presented with respect to their orders and to their time steps. The data reveal the following phenomena. First, the solutions of a high order approximation fall below those of a lower order approximation. Second, the first phenomenon does not hold in the solutions for various time steps with a fixed approximation order. Since the Runge-Kutta method did not offer any efficiency in the area of high order approximations, the finite difference method was considered. 
Equations (1.1) and $(1.2 a, b, c)$ were solved numerically using the finite difference method for both the flat plate and the sphere. The results for various time steps were presented. The table shows that the second phenomenon found in the Runge-Kutta method again occurs in the solutions generated by the finite difference method with respect to time step.

Finally, two comparsions were made of the numerical solutions. The first one was made between the Runge-Kutta method and the finite difference method. The results show that there is a good agreement between the two methods, and the differences between their solutions are, on the average, less than $3.1 \%$ in both cases. The second comparsion was made between the solution of a flat plate and that of a sphere. According to the finite difference method, the surface temperature of a flat plate decays much faster than that of a sphere.

To conclude this thesis, a numerical scheme is proposed as an alternative to the existing numerical methods. Recall the method of successive approximations described in chapter 2 . One of the major difficulties of that method is choosing the initial approximation for the iteration procedure. As mentioned, the convergence of the algorithm can be accelerated if one could obtain an initial approximation which is close to the exact solution. To determine this value, one could first use the the finite difference method (without the extrapolation algorithm) described in chapter 4 to determine the surface temperature. Then, by treating it as an initial approximation, the method of successive approximations is applied to obtain the solution. Undoubtedly, the temperature obtained using the finite 
approximation of the exact solution than the temperature at the previous time level. In addition, this technique would allow larger time steps. So far, nothing has been proved for this method, and the algorithm is left to the readers for both analytical and numerical justification. 


\section{References}

1. Saul S. Abarbanel, 'Time dependent temperature distribution in radiation solids', J. Math. Phys. 39, \#4, pp. 246-257(1960).

2. D. L. Ayers, 'Transient coolibg of a sphere in space', J. Heat Transfer, Feb. , pp. 180-181(1970).

3. A. L. Crosbie and R. Viskanta, "Transient heating or cooling of onedimensional solids by thermal radiation in Proceedings of the Third International Heat Transfer Conference, Vol. V, pp.146-153. A.I.Ch.E., New York(1966).

4. A. L. Crosbie and R. Viskanta, 'Transient heating or cooling of a plate by combined convection and radiation', Int. J. Heat. Mass. Transfer 11, pp. 345317(1968).

5. A. L. Crosbie and R. Viskanta, 'A simplified method for solving transient heat-conduction problems with nonlinear boundary conditions', J. Heat Transfer, Aug. , pp. 358-359(1968).

6. R. S. Fairall, R. A. Wells, and R. L. Belcher, 'Unsteady-state heat transfer in solids with radiation at one boundary', J. Heat Transfer, Aug. , pp. 266267(1962).

7. J. D. Lawson and J. L. Morris, 'The extrapolation of first order method for parabolic partial differential equations, I', SIAM Num. Analy. 17, pp. 641655(1980).

8. J. L. Milton and W. P. Goss, 'Stability criteria for explicity finite difference solutions of the parabolic diffusion equation with nonlinear boundary conditions', Int. J. Num. Meth. Engn. 7,pp. 57-67(1973).

9. J. L. Milton and W. P. Goss, 'On solving th etransient conducting slab with radiating and convecting surfaces', J. Heat Transfer, Nov., pp. 547548(1974).

10. D. U. von Rosenberg, Methods for the Numerical Solutions of Partial Differential Equations. American Elsevier Publishing Co. , New York, 1969, pp. $75-77$.

11. P. J. Schneider, 'Radiation cooling of finite heat-conducting solids', J. Aero/Space Science 27, pp. 548-549(1960).

12. S. D. Williams and D. M. Curry, 'An implicit-iterative solution of the heat conduction equation with a radiation boundary condition', Int. J. Num. Meth. Engn. 11, pp. 1605-1619(1977). 
13. Roberts and Kaufman, Table of Laplace Transforms. W. B. Saunders Co. , London, 1966.

14. V. Mangalis, Handbook of Series for Scientists and Engineers. Academic Press, New York, 1965, p. 5.

15. J. Todd, ed., A Survey of Numerical Analysis. McGraw-Hill, New York, 1962, pp. 322-323. 


\section{APPENDIX}

\section{THOMAS ALGORITHM}

The equations are:

$$
\mathrm{a}_{\mathrm{i}} \mathrm{U}_{\mathrm{i}-1}+\mathrm{b}_{\mathrm{i}} \mathrm{U}_{\mathrm{i}}+\mathrm{c}_{\mathrm{i}} \mathrm{U}_{\mathrm{i}+1}=\mathrm{d}_{\mathrm{i}}
$$

where $1 \leq i \leq N$ with $a_{1}=c_{1}=0$, and $N$ is the number of nodes in the domain.

The algorithm is as follows:

$$
\text { DO } \begin{aligned}
& 10 i=2, N \\
& \text { ratio }=a_{i} / b_{i} \\
& b_{i}=b_{i}-\text { ratio } * c_{i-1} \\
& d_{i}=d_{i}-\text { ratio } * d_{i-1}
\end{aligned}
$$

10 CONTINUE

$$
\begin{aligned}
& U_{N}=d_{N} / b_{N} \\
& \text { DO } 20 i=N-1,1 \\
& \quad U_{i}=\left(d_{i}-c_{i} * d_{i+1}\right) / b_{i}
\end{aligned}
$$

20 CONTINUE 